\title{
Exercise reprograms the inflammatory landscape of multiple stem cell compartments during mammalian aging
}

Ling Liu ${ }^{1,2,12}$, Matthew T. Buckley ${ }^{3,12}$, Jaime M. Reyes ${ }^{4,5,6,12}$, Soochi Kim ${ }^{1,2}$, Lei Tian, Mingqiang Wang ${ }^{7}$, Alexander Lieu ${ }^{1,2}$, Michelle $\mathrm{Mao}^{1,2}$, Cristina Mateo-Rodriguez ${ }^{1,2}$, Heather Ishak $^{1}$, Mira Jeong ${ }^{5}$, Joseph C. Wu ${ }^{7,8,9}$, Margaret A. Goodell ${ }^{4,5,6,13}$, Anne Brunet ${ }^{2,3,10,13}$, Thomas A. Rando ${ }^{1,2,11,13,14 *}$,

${ }^{1}$ Department of Neurology and Neurological Sciences, Stanford University School of Medicine, Stanford, CA, USA.

${ }^{2}$ Paul F. Glenn Center for the Biology of Aging, Stanford University School of Medicine, Stanford, CA, USA.

${ }^{3}$ Department of Genetics, Stanford University, Stanford, CA, USA.

${ }^{4}$ Stem Cells and Regenerative Medicine Center, Baylor College of Medicine, Houston, TX, USA.

${ }^{5}$ Department of Molecular and Human Genetics, Baylor College of Medicine, Houston, TX, USA.

${ }^{6}$ Department of Molecular and Cellular Biology, Baylor College of Medicine, Houston, TX, USA.

${ }^{7}$ Stanford Cardiovascular Institute, Stanford University, Stanford, CA, USA.

${ }^{8}$ Department of Medicine, Stanford University, Stanford, CA, USA.

${ }^{9}$ Department of Radiology, Stanford University, Stanford, CA, USA.

${ }^{10} \mathrm{Wu}$ Tsai Neurosciences Institute, Stanford University, Stanford, CA, USA.

${ }^{11}$ Neurology Service and Rehabilitation Research and Development Center of Excellence, Veterans Affairs Palo Alto Health Care System, Palo Alto, CA, USA.

${ }^{12}$ Equal contribution

${ }^{13}$ Senior author

${ }^{14}$ Lead contact

*correspondence to: rando@stanford.edu 


\begin{abstract}
Exercise has the ability to rejuvenate stem cells and improve tissue homeostasis and regeneration in aging animals. However, the cellular and molecular changes elicited by exercise have not been systematically studied across a broad range of cell types in stem cell compartments. To gain better insight into the mechanisms by which exercise affects niche and stem cell function, we subjected young and old mice to aerobic exercise and generated a single cell transcriptomic atlas of muscle, neural and hematopoietic stem cells with their niche cells and progeny.

Complementarily, we also performed whole transcriptome analysis of single myofibers from these animals. We identified common and unique pathways that are compromised across these tissues and cell types in aged animals. We found that exercise has a rejuvenating effect on subsets of stem cells, and a profound impact in the composition and transcriptomic landscape of both circulating and tissue resident immune cells. Exercise ameliorated the upregulation of a number of inflammatory pathways as well as restored aspects of cell-cell communication within these stem cell compartments. Our study provides a comprehensive view of the coordinated responses of multiple aged stem cells and niche cells to exercise at the transcriptomic level.
\end{abstract}




\section{INTRODUCTION}

Aging leads to stem cell dysfunction resulting in deterioration in tissue homeostasis and regeneration (Brunet and Rando, 2017; Chandel et al., 2016; Goodell and Rando, 2015; Rando and Wyss-Coray, 2014; Ren et al., 2017). Stem cell aging is manifested distinctly at the cellular and molecular levels in different tissues. For example, with age, neural stem cells (NSCs) are depleted in the brain hippocampus and subventricular zone (SVZ), hematopoietic stem cells (HSCs) skew towards the myeloid lineage, and skeletal muscle stem cells (MuSCs) exhibit increased susceptibility to cell death and delayed cell activation in response to injury (Gage and Temple, 2013; Jones and Rando, 2011; Liu and Rando, 2011; Navarro Negredo et al., 2020). In addition, aging elicits distinct alterations across the different cell types in a stem cell niche. Increased heterogeneity in gene expression has been found in various stem cells and the niche cells (Hernando-Herraez et al., 2019; Mahmoudi et al., 2019; Salzer et al., 2018; Xie et al., 2020). To delineate the complexity of aging at the stem cell, tissue and organismal level, it is critical to examine changes in the regulatory molecules and pathways simultaneously across cell types and tissues in aging animals.

Physical exercise has the health benefit of antagonizing the metabolic and immune changes that underlie various age-related diseases, lowering the mortality risk from cardiovascular diseases and cancer (Saint-Maurice et al., 2019). In patients with type II diabetics, physical exercise leads to significant reduction in the plasma Hemoglobin $\mathrm{A}_{1 \mathrm{c}}$ level, an indication of improved glycemic control (Sigal et al., 2007). In people with dyslipidemia, the amount of physical activity positively correlates with the degree of improvement in their 
lipoprotein profile (Kraus et al., 2002). Even in people with symptomatic but stable coronary artery disease, an increase in regular physical activity leads increased expression of total endothelial nitric oxide synthase (eNOS), enhanced vasodilatory responses to acetylcholine, and regression of atherosclerotic lesions (Hambrecht et al., 2003). Immunosenescence is a feature of aging and related to an increased susceptibility to infections, autoimmune diseases, chronic inflammation, cancer, and neurologic disorders. Recent evidence suggests that physical exercise has the ability to remodel the immune system during aging and therefore shape the immune landscape in later life (Nieman and Wentz, 2019). In addition, physical activity is associated with lower risk of dementia and neurodegenerative diseases including Alzheimer's disease and Parkinson's disease (Fang et al., 2018; Rovio et al., 2005; Scarmeas et al., 2009).

Increasing evidence indicates that physical exercise also has beneficial effects on stem cell function, tissue homeostasis and regeneration in adult animals. Running enhances neurogenesis and learning in the adult mouse brain, and counters the reduction of hippocampal neurogenesis during aging (De Miguel et al., 2021; Horowitz et al., 2020; van Praag et al., 2005). MuSCs in aged animals exhibit reduced expression of Cyclin D1 and a delay in activation in response to acute muscle injury (Brett et al., 2020). Exercise increases Cyclin D1 expression and enhances muscle regeneration in old animals (Brett et al., 2020). A recent study identifies a subset of osteogenic progenitors in the bone marrow as niche cells for the common lymphoid progenitors (CLPs) which diminish with age (Shen et al., 2021). Voluntary running increased the number of both the osteogenic progenitors and CLPs in aging animals without affecting the frequency of the HSC population (Shen et al., 2021). However, up to date, studies on the mechanisms by which exercise modulates stem cell function have generally not included effects of exercise on local niche cells or endocrine influence from distal cells that are not in direct 
contact with stem cells. It remains unclear how niche cells of various stem cell compartment are affected in ways that can exert a combinatorial influence on stem cell function following exercise. It is also largely unknown how changes induced by exercise in skeletal muscle, the direct target and beneficiary of physical exercise, are mechanistically connected to changes in distal tissues and the overall health of an individual. A comprehensive examination of the molecular changes induced by exercise across tissue and cell types is critical to understand the systemic effect of exercise in reshaping the molecular and cellular processes in stem cells and their niche environment to improve tissue homeostasis and regeneration during aging.

The development of single cell RNA sequencing (scRNA-seq) technology has led to the creation of atlases of tissues, including those from aged animals, which report changes in the transcriptome of individual cells across multiple tissues and organs (Martinez-Jimenez et al., 2017; Tabula Muris, 2020). The effect of caloric restriction on the cellular composition and transcriptional network of aged tissues has also been examined at the single cell level (Ma et al., 2020). It remains unknown whether other forms of aging intervention exert similar systemic effect across tissues as caloric restriction. Changes in the interaction between stem cells and their niche during aging and in response to aging interventions have not been explored. In this study, we generated an integrative single cell atlas to understand the effect of exercise in modulating stem cell function and tissue homeostasis during aging. We systematically analyzed 435,628 single cells from skeletal muscle, the SVZ of the brain, bone marrow HSCs and peripheral blood immune cells of young and old mice with and without a period of voluntary running exercise. We analyzed cell type composition changes, transcription changes and intercellular communication changes in these stem cell compartments. Although exercise induced a much smaller subset of genes in aged animals, it was able to ameliorate the elevated inflammation in 
cell types from all these stem cell compartments. Computational analysis of predicted ligandreceptor interaction also suggests that intercellular communication can be restored by exercise. In addition, we have performed bulk RNA-seq to understand gene expression changes in single muscle fibers, the contractile units that not only generate the movements that are the basis of exercise-mediated effects, but are also the key niche cell of MuSCs. We find that exercise largely erases the age-difference in the transcriptome of muscle fibers. Overall, our study provides a comprehensive molecular framework for the effect of exercise in modulating cellular and molecular processes during aging. 


\section{RESULTS}

\section{Construction of aging and exercise mouse single-cell atlases by scRNA-seq}

To understand the transcriptional changes induced by aerobic exercise in stem cells and their associated niche cells in muscular, neural, and hematopoietic systems of young and aged animals, we housed young ("Y"; 4 months of age) and old ("O"; 22 months of age) male C57BL/6J mice in cages in which rotating running wheels were attached to digital recorders (Figure 1A). Age-matched control mice were housed in identical cages in which the wheels were removed. All mice provided with the running wheels adapted to a voluntary exercise routine during the dark phase of the sleep-wake cycle within one week of exposure. We harvested skeletal muscle, the subventricular zone (SVZ) of brain, bone marrow, and blood from these exercised and control mice 5 weeks later. We processed the tissues into single-cell suspensions and eliminated dead cells by FACS. Due to the rarity of HSCs in the bone marrow, we used a cocktail of antibodies against surface markers of HSCs to enrich this population. Live single cells were then captured and a total of 458,754 cells from the 4 tissues were sequenced. After quality control, we retained 435,628 cells in our single-cell atlas for downstream analysis.

We annotated cell clusters based on the expression levels of canonical cell-type-specific markers in each tissue (Figure S1A-D). Notably, while Pecaml ${ }^{+}$endothelial cells (ECs) and Acta2 $^{+}$smooth muscle cells from skeletal muscle and the SVZ (where they are referred to as mural cells) formed distinct clusters, immune cells from all 4 tissues clustered together (Figure 1B). All annotated cell types were present in the 4 experimental groups, control young mice (YC), exercised young mice (Y-Ex), control old mice (O-C) and exercised old mice (O-Ex) (Figure $\mathrm{S} 1 \mathrm{E})$. In total, we identified 14 types of cells in skeletal muscle, 15 in the SVZ, 11 in the 
periphery blood, and 6 in the HSC population (Figures 1C, E and S1F). Among all cell types, activated neural stem cells (aNSCs) and neuroblasts exhibited the most significant reduction with age, and exercise expanded these two populations of cells in both young and old animals (Figure $1 \mathrm{C}$ and D), supporting previous reports of the beneficial effect of exercise on adult neurogenesis (van Praag et al., 1999; van Praag et al., 2005). In the HSC compartment, aging was associated with an expansion in the long-term HSCs (LT-HSCs) (Figure 1F and G). Exercise was associated with a shift of cell expansion toward the myeloid multipotent progenitors (MyMPPs) and this effect was retained in exercised old animals (Figure 1F). The vast majority of MuSCs in adult animals reside in quiescence in skeletal muscle. Although neither aging nor exercise significantly altered the cell cycle profiles or the total number of MuSCs, we found that subsets of MuSCs were selectively diminished during aging (Figure 1G). These subsets of MuSCs were not defined by their differential expression of myogenic genes but by genes in metabolic and immune functions (Figure 1H and 1I). These data indicate that aging leads to a change in the number or the relative ratio of subsets of stem cells, and exercise partially reverse cell composition changes in old mice.

\section{Effect of aging on the transcriptomes of stem cells and niches cells}

To unveil common and unique gene expression changes associated with aging and exercise in skeletal muscle, brain, and the hematopoietic system, we identified differentially expressed genes (DEGs) from 22 major cell types in young control (Y-C), old control (O-C), young exercise (Y-Ex) and old exercise (O-Ex) mice (Table S1). Upregulated and downregulated DEGs in O-C when comparing to Y-C mice are defined as "age-DEGs". We found that cells in the muscle were less impacted by aging based on the number of age-DEGs in each cell type 
(Figure 2A). Endothelial cells, smooth muscle cells (SMCs), monocytes, B and T cells from the muscle all manifested smaller numbers of age-DEGs to their counterparts in other tissues. The most striking example is monocytes. We identified 960 age-DEGs in the monocytes from the blood, the most among all 22 cell types. On the contrary, we identified 257 age-DEGs in the monocytes in aged muscle. Common age-DEGs in the same cell types between different tissues varied from $7.3 \%$ to $21.8 \%$ (Figure 2B). However, despite the distinct identity of age-DEGs in the same cell type from different tissue, these age-DEGs were implicated in similar biological pathways as exemplified by endothelial cells from the muscle and the SVZ (Figure 2C).

To gain insight into the common and unique genes impacted by the aging process in these tissues, we separately compared upregulated and downregulated age-DEGs in HSCs and in major immune cell types in the peripheral blood, the muscle, and the SVZ. We identified 4 common upregulated and 12 common downregulated age-DEGs in HSCs and immune cells in peripheral blood (Figure S2A and S2B). An additional 19 downregulated age-DEGs were found in the circulating immune cells (Figure S2B). Among the 31 downregulated age-DEGs in the immune cells, 6 were ribosome genes. The major cell types in the SVZ shared 57 common upregulated and 10 common downregulated age-DEGs (Figure 2D, S2C). Among the 56 upregulated age-DEGs, 17 encode subunits of enzymes that play crucial roles in oxidative phosphorylation (Figure S2D and S2E). These enzymes, including ubiquinol-cytochrome c reductase (UQCR), cytochrome c oxidase (COX), NADH:ubiquinol oxireductase (NDUF) and ATP synthase, have been implicated in a number of neurodegenerative diseases such as Parkinson's and Alzheimer's disease (Manczak et al., 2004). In addition, 20 genes encoding ribosomal proteins or ribosome-associated proteins were found upregulated in SVZ cells from aged animals. It is worth noting that although no one single gene changes expression across all 
cell types in these 3 tissues, genes encoding various members of the heat shock protein 90 family were consistently found in the common downregulated age-DEGs (Figure 2E).

We have also explored the biological implication of age-DEGs from the 21 major cell types in skeletal muscle, the SVZ, and peripheral blood/bone marrow using gene set enrichment analysis (GSEA). We found that although different cell types from different tissues exhibited a high degree of variability in age-DEGs, the cellular functions and responses in which these DEGs are implicated converge into a set of specific GSEA hallmark and KEGG pathways. The upregulated age-DEGs across cell types clearly revealed that aging is associated with an elevated inflammatory systemic environment. The age-DEGs from all 21 major cell clusters in our analyses indicated an increase in the interferon gamma (IFN $\gamma$ ) response, and the age-DEGs from 18 out of the 21 cell clusters indicated an increase in the interferon alpha (IFN $\alpha)$ response (Figure 2F). The majority of cell types from the muscle and peripheral blood/bone marrow exhibited an elevated response in interleukin 2 (IL2), IL6 and tumor necrosis factor $\alpha$ (TNF $\alpha$ ) signaling which were absent in the majority of SVZ cells. Contrary to most of the cell types, peripheral monocytes appeared to manifest a downregulation in TNF $\alpha$ signaling (Figure 2E). Strikingly, the expression of ribosomal proteins exhibited noteworthy changes with age. Significant upregulation of ribosomal genes with age was found in the majority of SVZ cells and in the majority of non-immune cells in skeletal muscle, whereas immune cells as well as HSCs downregulated the expression of ribosomal genes with age (Figure 2G). Metabolically, a significant increase in oxidative phosphorylation and, to a less extent, glycolysis with age was observed in SVZ cells but not muscle cells. Similarly, a significant decrease in MYC target gene expression and G2M checkpoint genes with age was found in the SVZ and hematopoietic system but not in muscle, including stem cells in these tissues, consistent with changes of cell cycle 
profiles in the NSCs and HSCs but not MuSCs. Changes unique to muscle resident cells also included a decrease in the interaction with extracellular matrix (ECM) (Fig. 2G).

\section{Reversal of age-induced gene expression changes by exercise}

We next explored the effect of exercise on gene expression patterns of various cell types across tissues and with age. Upregulated and downregulated DEGs in exercised mice when comparing to control mice of the same age are defined as "exercise-DEGs" (Ex-DEGs).

Downregulated age-DEGs that increase expression and upregulated age-DEGs that decrease expression in response to exercise are collectively termed "restored genes". A much smaller set of Ex-DEGs were identified in most of the cell types from old animals compared to young animals, with notable exceptions in FAPs, immune cells from muscle, and HSCs (Figure 3A). Among the stem cell types, HSCs exhibited the smallest set of Ex-DEGs. A higher number of Ex-DEGs were induced in various immune cell types in the muscle, whereas a much lower number of Ex-DEGs were induced in the immune cells in peripheral blood from old animals. In young animals, monocytes from peripheral blood exhibited the largest set of Ex-DEGs. In old animals, monocytes from muscle exhibited the highest number of Ex-DEGs which included 77\% of Ex-DEGs identified in muscle monocytes from young mice (Figure S3A). Common Ex-DEGs in the same cell types between different tissues varied from $2.6 \%$ to $8.5 \%$ (Figure S3B). Muscle cells exhibited higher ratio of restored genes in comparison to cells from the other tissues (Figure 3B).

We then performed pathway analyses with Ex-DEGs and found that exercise induced genes encoding metabolic enzymes implicated in oxidative phosphorylation in the majority of cells in young animals, including all cell types in the SVZ. In contrast, upregulation of these 
genes was almost absent in old animals (Table S1). Our analyses also suggested that a number of cytokine signaling pathways were suppressed by exercise (Table S1). In young animals, the downregulation of genes implicated in TNF $\alpha$ signaling was found in all non-immune cell types in muscle, all hematopoietic cells, and endothelial cells in the SVZ, most of which also exhibited evidence of downregulation in TNF $\alpha$ signaling in old animals. Genes implicated in IFN $\alpha$ and IFN $\gamma$ signaling were also found to be downregulated in some cell types from young animals following exercise. Interestingly, suppression of IFN $\alpha$ and IFN $\gamma$ signaling was found in immune cell types in muscle after exercise only in old animals. Exercise was not associated with expression changes of genes related to immune pathways in SVZ cell types with the exception of endothelial cells and microglia. In stark contrast, peripheral monocytes exhibited a downregulation of almost all immune signaling pathways found to be elevated in aged cells (Figure 3C).

We next explored the biological implications of exercise-restored genes in aged animals. As predicted from the overall higher ratios of restored genes in muscle cell types, nearly all cell types in muscle exhibited reversal of cellular signaling pathways and processes that changed during aging (Figure 3D). While the restored genes in non-immune cell types primarily related to tissue specific functions as exemplified by the enrichment of restored genes in pathways of epithelial-to-mesenchymal transition and myogenesis, restored genes identified in immune cells in muscle were enriched in cytokine signaling pathways. Consistent with the small number of Ex-DEGs identified in the majority cell types in the SVZ and hematopoietic systems (Figure 3A), exercise restored functional pathways that were altered in aged cells only in a limited number of SVZ and hematopoietic cell types (Figure 3D). Despite the fact that IFN $\alpha$ signaling and IFN $\gamma$ signaling were found to be elevated in virtually all cell types with age, exercise 
restored IFN signaling only in immune cell types in old animals. On the contrary, TNF $\alpha$ signaling was restored by exercise in cell types in all three tissues which exhibited changes in this signaling pathway during aging. Endothelial cells from both muscle and the SVZ restored the expression of cellular communication network factor 1 (CCN1) (Figure 3E), a secreted protein that promotes the adhesion of endothelial cells and angiogenesis. Exercise restored IL1b expression in monocytes and CCL5 expression in circulating T cells in old mice. These secreted factors may mediate broad effect of exercise on multiple tissues and cell types in a paracrine and endocrine manner.

To determine whether the effect of exercise on regulating the cellular inflammatory state was limited to the abovementioned pathways, we calculated an inflammatory score for each cell type based on the expression of selected genes in the GO term "inflammatory response", and compared this score of each cell type between the experimental conditions. We found that in young mice, exercise was associated with a lower inflammatory score in nearly all cell types in the muscle and the SVZ with the notable exception of macrophages from the muscle (Figure $3 \mathrm{~F}$ and S3B-D). In old mice, the correlation between a lower inflammatory score and exercise was still present in most of these cell types albeit the difference was smaller than that in the young animals. In addition, although no difference was found in the inflammatory score of HSCs in young animals, the inflammatory score of HSCs in the O-Ex mice was lower than that in the O-C mice. These analyses suggest that exercise has a broad effect on the overall inflammatory landscape of stem cells and their niche environment.

\section{Cell-cell communication network in skeletal muscle}


To examine the impact of aging and exercise on the intercellular communication network, we calculated the number of ligand-receptor interacting pairs and their interaction strength between each two-cell pairs within a tissue to test for paracrine and autocrine signaling. In skeletal muscle of young animals, we identified a total of 65 potential signaling pathways mediated by secreted or cell surface signaling molecules (Figure 4A). In addition, we identified 8 potential pathways mediated by extra-cellular matrix (ECM) components (Figure 4B). The strongest interaction was found between FAPs and tenocytes (Figure 4C). In comparison to other cell types in muscle, these two types of cells express high levels of ECM proteins and contributed the most to interactions mediated by ECM components in the muscle (Figure 4D). Terminal Schwann cells, located at the neuromuscular junction, contributed the most to the intercellular interaction mediated by secreted or cell surface factors whereas muscle-resident macrophages received the most secreted and cell surface signals (Figure 4E). Interactions between each two-cell pairs in the muscle of young mice are summarized in Table S2. A number of signaling pathways have been implicated in regulating MuSC homeostasis and function but the sources of ligands for these pathways thus far remain incompletely defined (Conboy et al., 2003; Pawlikowski et al., 2017; Ratajczak et al., 2003). Our analysis revealed the identity of cells that secret many of these ligands. For example, the ligands for the NOTCH pathway were contributed by endothelial cells, FGF signaling was from FAPs, Oncostatin M (OSM) signaling was initiated by monocytes, and CXCR signaling was initiated by SMC (Figure S4A). Thus, the intercellular network analysis provided a blueprint of the complex signaling network between niche cells and MuSCs in healthy young animals, and the foundation to study the cell-cell interaction changes that occur during aging and in response to exercise. 
We then compared the interaction strength between each two cell types from young and old mice, and from old mice before and after exercise. With age, intercellular communication was diminished in interactions initiated by Schwann cells, tenocytes, and T cells, and to a lesser degree, endothelial cells, whereas the interactions initiated by other cell types appeared generally enhanced (Figure S4B, left). Exercise restored the interaction initiated by Schwann cells, endothelial cells and FAPs (Figure S4B, right). In total, we identified 38 signaling pathways that changed with age, half of which were restored or partially restored after exercise (Figure S4C). Among these 38 pathways, 10 were involved in the interaction between MuSCs and other niche cell types (Figure 4F). PTN signaling between MuSCs and Schwann cells and tenocytes was reduced in old mice regardless of the exercise status. FGF signaling between MuSCs and FAPs was diminished due to the downregulation of Fgf18 expression in FAPs in old mice. Consistent with the elevated inflammation associated with aging as previously discussed, CXCL signaling between MuSCs and signal-sending cells was upregulated with age but not restored by exercise (Figure 4F). On the contrary, OSM signaling and NOTCH signaling were diminished in MuSCs from old animals and were restored by exercise, whereas the signaling between Midkine (MDK) and Syndecan 4 (SDC4) receptor, which has been implicated in inflammatory response (Sorrelle et al., 2017), was induced during aging and abolished by exercise (Figure 4F). In addition, exercise was associated with the activation of PROS and GAS signaling in MuSCs in old animals, likely due to the induction of AXL receptor expression (Figure 4G). It is worth noting that exercise was associated with AXL expression also in macrophages in old animals. The expression of AXL ligands, PROS1 and GAS6, was diminished in macrophages from control old animals and restored in exercised old animals. As the activation of AXL signaling has been shown to have immune suppression function in tumor microenvironment (Tanaka and Siemann, 
2020), it will be interesting to determine whether the activation of PROS and GAS in O-Ex mice plays a role in regulating MuSC function by suppressing inflammation in the local niche. As muscle monocytes exhibited the largest number of Ex-DEGs among all cell types in old animals (Figure 3A), we determined whether this robust gene expression change is linked to restoration of monocyte interaction with other cell types in the muscle. We found that the increase in Illb expression and the loss of Osm expression with age were reversed in exercised old mice (Figure 4H). The expression of a number of receptors that exhibited reduced or diminished expression with age was also restored with exercise (Figure 4I and J). These included FPR1 and FPR2, which are receptors for Annexin 1 (ANXA1), Integrin $\beta 21$ which binds to Junction Adhesion Molecule 2 and 3 (JAM2 and JAM3), and Integrin $\alpha$ which binds to THY1, JAM2 and JAM3. ANXA1 is a phospholipid binding protein that has anti-inflammatory activity (Perretti and D'Acquisto, 2009). JAM2 and JAM3 regulate cell adhesion and migration (Kummer and Ebnet, 2018). These findings suggest that exercise has an immune modulating effect in skeletal muscle, and intercellular interactions in the MuSC niche were largely restored to youthful levels by exercise.

\section{Cell-cell communication network in the SVZ}

In the SVZ of young animals, we identified a total of 52 potential signaling pathways mediated by secreted or cell surface signaling molecules (Figure 5A). In addition, we identified 6 potential signaling pathways mediated by ECM components (Figure 5B). The strongest stem cell-niche cell interaction was identified between NSCs and OPCs (Figure 5C). Mural cells are the major initiator of ECM interactions (Figure 5D), whereas OPCs mediated the most interactions by secreted and cell surface proteins (Figure 5E). Interactions between each two-cell 
pairs in the SVZ of young mice are summarized in Table S3. Strong interactions and a high degree of signaling similarity were found between the cell types of ectodermal origin including qNSCs, aNSCs, Neuroblasts, OPCs and oligodendrocytes, whereas cells of the mesodermal origin, including mural, microglia and endothelial cells, exhibit similar signaling patterns (Figure S5A). Although a high degree of signaling similarity was found between the neural cell types, we found that the qNSCs and aNSCs exhibited distinct signaling patterns with cells in the SVZ niche. For example, qNSCs expressed WNT7b and interacted with the Frizzle receptors on aNSCs, OPCs and endothelial cells (Figure S5B), whereas aNSCs expressed EFNA2 and EFNA5 and interacted with EPH receptors on themselves, OPCs, and, to a lesser degree, qNSCs, neuroblasts and endothelial cells. This cell-cell interaction analysis provides valuable insights into the common and distinct signaling pathways that regulate NSCs and various niche cell types.

Overall, aging weakened the intercellular communication in the SVZ (Figure 5F, left). Neuroblasts and aNSCs exhibited a decrease in their interaction with all types of SVZ cells in old animals. This decrease is attributable to the depletion of aNSCs and neuroblasts with age (Figure 1D), as the cell communication calculation factors in the effect of population size on interaction strength. Consistent with this, the expansion of aNSCs and neuroblasts coincided with an increase in their interaction with all types of SVZ cells in O-Ex mice (Figure 5F, right). We identified 27 signaling pathways that changed with age in the SVZ, 13 of which were restored or partially restored after exercise (Figure S5C). Our analysis revealed that a number of SVZ cell types interacted with endothelial cells via TWEAK signaling (Figure 5G and $\mathrm{H}$ ). Aging was associated with a loss of Tnfrsf $12 a$ expression in endothelial cells and lack of TWEAK signaling in the SVZ (Figure 5G and I). Exercise was associated with the restoration of Tnfrsf12a 
expression in endothelial cells and thus TNFSF12 signaling (Figure S5F and G). TNFSF12 has been found to promote the proliferation and migration of endothelial cells and thus a proangiogenesis cytokine (Lynch et al., 1999; Wiley et al., 2001). Our findings suggest that it may be a key mediator of the beneficial effect of exercise on vascular health in the brain. On the contrary, the interaction between microglia and a number of SVZ cell types was enhanced in old nice (Figure 5F, left) and was further elevated by exercise (Figure 5F, right). Microglia in O-C animals gained active BAFF, THY1 and TNF signaling (Figure 5J). The activation of these pathways was due to the expression of genes encoding TNFSF13b and TNF by microglia and THY1 by aNSCs (Figure 5K). Exercise was associated with an abolishment of Thyl expression by aNSCs (Figure S5I). However, the expression of Tnfsf13b and Tnf in microglia was not affected by exercise in old animals. Interestingly, while TNFSF13b and TNF are generally believed to be pro-inflammatory, exercise of old animals was associated with the endothelial expression of the anti-inflammatory cytokine Cx3c11 (Figure S5C and D), resulting in the activation of $\mathrm{CX} 3 \mathrm{C}$ signaling in microglia. In addition, exercise was associated with OPC expression of L1 Cell Adhesion Molecule (L1Cam), an adhesion molecule that plays an important role in the development of the nervous system (Schafer and Frotscher, 2012), and Angiopoietin Like 1 (ANGPTL1), which regulates glucose homeostasis and lipid metabolism (Oike et al., 2005). Taken together, these data reveal potential mechanisms by which exercise mitigates the decline of brain health by reprogramming the cell-cell communication network in the neurogenesis region.

\section{The effect of aging and exercise on immune cells}


To understand the effect of aging and exercise on immune cells, we first compared the fraction of major immune cell types in the 4 groups of animals. We found that B cells remain fairly constant with age and in response to exercise (Figure S6A and S6B). We annotated a number of subtypes of $\mathrm{T}$ cells and found a 4-fold reduction in the percentage of $\mathrm{CD} 4^{+} \mathrm{T}$ memory cells of all $\mathrm{T}$ cells in O-C mice (Figure 6A and S6B). With age, a 12\% reduction was found in the percentage of Treg cells, whereas the fraction of $\mathrm{CD}^{+} \mathrm{T}$ cells exhibited a 2 -fold increase. Exercise was associated with a 56\% and 54\% increase in the Treg fraction in both young and old animals, respectively. The fraction of NK cells was reduced more than 2-fold with age but was restored with exercise. Monocytes exhibited a robust increase with age and in response to exercise, however, the percentage of Ly6c ${ }^{+}$and Ly6c ${ }^{-}$monocytes differs (Figure 6B). Given the immunosuppressive nature of Treg cells and their function in limiting chronic inflammatory diseases and that Ly6c $\mathrm{c}^{-}$monocytes preferentially differentiate into M2 macrophages (Olingy et al., 2017), our data suggest that aging has a profound impact on the composition of leukocyte subtypes, and that exercise steers the composition of immune cells towards an anti-inflammatory phenotype.

We identified 34 pathways mediated by secreted or cell surface proteins among the immune cells in the peripheral blood (Figure 6C). While subtypes of T cells share high degree of similarity in both outgoing and incoming signals, B cells share similar intercellular communication patterns with natural killer cells, monocytes, neutrophils and dendritic cells (Figure S6C). Based on the sum of signaling strength in all blood cells, we identified 19 signaling pathways that changed in O-C mice in comparison to $\mathrm{Y}-\mathrm{C}$ animals (Figure S6D). Unlike in the muscle and SVZ where a majority of intercellular interaction that changed with age were restored in O-Ex animals, only a small number of pathways were restored among blood 
cells in O-Ex animals. Treg cells were the only T cell subtype that expressed CD39 (ENTPD1) in Y-C mice (Figure 6D). CD39 is a membrane nucleotidase that hydrolyzes extracellular ATP to generate adenosine. The CD39-adenosine axis has an immune suppression effect on effector T cells and NK cells (Deaglio et al., 2007). CD39 expression was diminished in old animals (Figure 6E). The reduction in both the number and the immune suppressive activity of Treg cells is consistent with an overall elevated inflammation status in old animals. Galectin 9, encoded by the gene Lgals9, is an S-type lectin that induces the differentiation of naïve T cells into Treg cells and maintains a proper pool of Treg cells in animals (Seki et al., 2008). We found that Lgals9 expression in Treg cells declined with age but was restored by exercise in old animals (Figure $6 \mathrm{~F})$, consistent with the pattern of change in Treg cell number. Although the mechanism by which exercise induces the expression of $\operatorname{Lgals} 9$ remains to be determined, these analyses suggest that Treg cells may play an important role in mediating the immune modulating effect of exercise. We also compared the signaling patterns between the two subpopulation of monocytes based on Ly6c2 expression. The analysis revealed that although Ly6c $2^{+}$monocytes were more active in cell-cell signaling than Ly6c2- monocytes in Y-C mice, Ly6c2- monocytes became more active in response to exercise (Figure 6G). Understanding the mechanisms by which these subpopulations of circulating $\mathrm{T}$ cells and monocytes change their signaling networks will provide insights in how exercise affects the immune system.

We found changes in the expression of a number of signaling molecules universally in all immune cell types. One example is Platelet Factor 4 (Pf4). We found that Pf4 was expressed in Ly6c2+ monocytes and neutrophils in Y-C mice, but induced in all other cell types in old animals (Figure 6H). This raised an interesting question whether the change in Pf4 expression could be traced back to HSCs and hematopoietic progenitors. 


\section{Aging and Exercise-induced Changes in Myofibers}

Not only are muscle fibers the structural basis for mobility during exercise, but they also secret circulating factors termed myokines that can affect distant tissues and cells (Pedersen and Febbraio, 2012). However, due to the multinucleated nature of muscle fibers, they are excluded from the scRNA-seq analysis. To understand the effect of aging and exercise on muscle fibers, we isolated individual muscle fibers free of attaching cells from the 4 groups of mice and performed RNA-seq analysis (Figure 7A). Although Y-C and O-C fibers were transcriptionally distinct, Y-Ex and O-Ex fibers shared a high degree of similarity in their transcriptomes (Figure 7A). The most prominent function of the DEGs that distinguished the fibers from exercised and control animals regardless of age was in the insulin signaling pathway (Figure S7A). Exercise led to changes in distinct metabolic and immune pathways in animals of different ages. In young animals, Ex-DEGs were implicated in the citrate cycle, unsaturated fatty acid biosynthesis, and IFN $\gamma$ response, whereas in old animals, Ex-DEGs were implicated in glycerophospholipid metabolism and TGF $\beta$ signaling (Figure S7B). We found $24 \%$ of the downregulated age-DEGs restored in myofibers of O-Ex animals. The most prominent pathway that these restored genes implicated in was the lipid metabolic process (Figure 7B). Among the upregulated age-DEGs, 15\% were restored, including those implicated in TNF $\alpha$ signaling pathway (Figure 7C).

To determine how muscle fibers modulate the local niche environment for MuSCs and the systemic milieu that may signal distant tissues and cells, we compared the RNAseq output data to a comprehensive database of ligand-receptor interaction (Shao et al., 2021), and identified age-DEGs and Ex-DEGs encoding secreted proteins (Figure 7D and S7C). Exercise induced the expression of a larger number of secreted protein in young animals in comparison to old animals 
(Figure S7C). The fold change exhibited by these Ex-DEGs encoding secreted proteins was also greater in young animals. We found that exercise decreased the expression of cytokine genes that were upregulated with age such as $C x c l 10$, and increased the expression of a number of growth factors, such as Insulin Growth Factor 1 (IGF1), Secreted Phosphoprotein 1 (SPP1) and Fibroblast Growth Factor 2 (FGF2), which were downregulated with age (Figure 7D). The increase in Sppl expression in myofibers was consistent with our previous intercellular interaction analysis that SPP1 signaling was induced in nearly all types of muscle resident cells in O-Ex mice (Figure S4C). Sppl has been shown to be a downstream target of Peroxisome Proliferation Activator Receptor $\gamma$ Coactivator $1 \alpha(\mathrm{PGC}-1 \alpha)$ to activate macrophages and enhance angiogenesis in skeletal muscle (Rowe et al., 2014). We used the NicheNet cell-cell interaction package to identify receptors and target genes of SPP1 in muscle resident cells (Browaeys et al., 2020). Our analysis predicted that receptors of SPP1 are expressed in all major cell types in muscle (Figure 7E), and that exercise was associated with the reversal of the change in the expression of the majority of predicted SPP1 target genes across muscle cell types (Figure 7F and S7D). These reversed SPP1 target genes include cytokine genes Ccl11, Ccl3, Illb, Csf1, Btg2, and genes encoding transcription factors, such as Irf8 and Stat1, which play important roles in regulating immune function (Figure S7D). Furthermore, GSEA analysis of these SPP1 target genes that reversed their expression in exercised mice suggests that they were involved in TNF $\alpha$ and IL2 pathways in a number of cell types (Figure 7G). Taken together, these data suggest that myofibers produce cytokines and growth factors in response to exercise to modulate the immune profiles of muscle resident cells in old animals. 


\section{DISCUSSION}

Tissue atlases at the single cell transcriptome level have been generated across the lifespan of mammalian species in recent years (Ma et al., 2020; Martinez-Jimenez et al., 2017; Tabula Muris, 2020). These atlases have provided unprecedented resolution on cell composition and transcriptomic changes in a broad range of tissues in aged animals. However, due to their rarity, stem cells are often not identifiable in these atlases, or are present at a frequency that is too low to reveal statistically significant changes between age groups. Our study set out to understand the cell type composition changes in stem cell compartments and the molecular changes that occur in stem cells and niche cells during aging and how exercise as an aging intervention modulates these changes. Towards this end, we generated a single cell transcriptomic atlas with sufficient representation of HSCs, MuSCs and NSCs, as well as their surrounding niche cells, from young and old mice subjected to voluntary exercise or sedentary housing. This work complements existing atlases in expanding our knowledge on the systemic changes that occur during aging, and provides a wealth of knowledge in understanding the physiological effect of exercise at the cellular and molecular levels.

To ensure that the multinucleated myofibers, the cells responsible for locomotion, were included in our transcriptomic analysis, we also performed RNA-seq with single myofibers that are devoid of neighboring mononucleated cells. Accompanying the development of scRNA-seq technologies, computational tools have also been developed to infer changes in cell type composition and in the transcriptome of a defined cell type from whole tissue bulk RNA-seq data (Newman et al., 2019). The combination of our scRNA-seq with skeletal muscle, the single fiber RNA-seq and existing bulk RNA-seq data from the entire muscle provides an ideal dataset to 
develop and test such computational tools. Skeletal muscle is the largest organ of the mammalian body. In addition to the contractile function, increasing evidence has indicated that skeletal muscle is also a secretory organ in response to exercise (Pedersen and Febbraio, 2012). However, the exact cellular source for many exercise-induced cytokines and growth factors in muscle tissue remains unclear. The datasets generated in our study provide a foundation to distinguish myokines, defined as cytokines secreted by myofibers (Pedersen and Febbraio, 2012), from those secreted by muscle resident cells. Understanding the regulation of these secreted proteins will provide valuable insights in the molecular mechanisms by which myofibers mediate the effect of exercise on muscle resident cells and distal tissues.

Previous scRNA-seq studies have provided compelling evidence that mammalian aging is associated with alterations in the immune system and in inflammatory responses. Manifestations of such changes include increased transcriptional variability during naïve T cell activation, infiltration of immune cells into the tissues where they are absent in young animals, and clonal expansion of lymphocytes (Dulken et al., 2019; Martinez-Jimenez et al., 2017; Tabula Muris, 2020). Consistent with findings from these studies, our scRNA-seq data reveal an increase in the expression of IFN responsive genes in all cell types and an increase in the expression of TNF $\alpha$ target genes in the majority of cell types from aged animals. In exercised old animals, the increased expression of genes in the IFN pathway is reversed in many cell types in the muscle and blood, and the increased expression of genes in the TNF $\alpha$ pathway is reversed in various cell types of the muscle, SVZ and blood. These findings provide strong evidence that exercise, similar to caloric restriction which has been shown in many species to slow organismal aging (Fontana and Partridge, 2015), represses the increase in inflammatory responses associated with aging. 
The single cell atlases generated by us and others provide direct evidence that exercise and caloric restriction, two aging interventions that have obvious metabolic effects on organismal health, modulate the inflammatory status of a broad range of cells and tissues (Ma et al., 2020). The role of the immune system in regulating cellular and organismal metabolism has been studied in the context of obesity. The infiltration of monocytes/macrophages and chronic inflammation in white adipose tissues have been shown to precede the significant increase in circulating insulin levels in a mouse models of genetic and high fat diet induced obesity (Xu et al., 2003). Mice defective in the TNF pathway are partially protected from obesity induced insulin resistance (Uysal et al., 1997). Our study reveals a reversal in the elevated expression of genes in the TNF pathway across multiple cell types and tissues, as well as a reversal of nearly all inflammatory pathways that are upregulated in monocytes in exercised old animals. Future studies on the functional outcome of these transcriptomic changes in monocytes may hold the key to understand the benefit of physical exercise in reducing the risk of metabolic syndromes.

Unlike cells from the muscle and blood, most cells in the SVZ of old animals, with the exception of microglia and endothelial cells, exhibit elevated IFN but not IL6 or TNF $\alpha$ signaling. We also find that the age-induced upregulation of TNF $\alpha$ and IL6 target genes in microglia can be rescued by exercise while the upregulation of IFN signaling persists. These analyses support the previous finding that infiltrating $\mathrm{T}$ cells in the brain secrete IFN $\gamma$, and suggest that exercise does not lead to the elimination of these infiltrating T cells. Interestingly, while the induction of IFN signaling in microglia in aged animals is not ameliorated by exercise, the expression of the cytokine gene $C x 3 c l 1$ is induced by exercise in old animals. Active CX3CL1 signaling is generally regarded as anti-inflammatory, and has been shown to suppress neuronal death in cocultures of neurons with IFN $\gamma$-stimulated microglia and in mouse models of Alzheimer's disease 
(AD) (Bhaskar et al., 2010; Cho et al., 2011; Mizuno et al., 2003). Deficiency of Cx3cl1 expression has also been found in brain specimen from AD patients (Cho et al., 2011). Whether active CX3CL1 signaling mediates the beneficial effect of exercise in reducing the risk of neurodegenerative conditions, including $\mathrm{AD}$, by limiting the neurotoxicity resulting from abnormal microglia activation requires further study.

The effect of physical exercise on the immune system is complex. The immediate changes in immune responses following exercise vary based on the intensity and duration of effort (Nieman and Wentz, 2019). Although frequent, heavy exertion appears to be linked to increased risks of upper respiratory tract infections in athletes (Nieman et al., 1990), there is compelling evidence that regular exercise at intermediate intensity confers health benefits to the immune system (Nieman and Wentz, 2019). The duration of voluntary running in our study was designed to model habitual aerobic exercise. We find that exercise exerts distinct effects on the immune system in animals of different ages. Exercise induces significantly larger number of DEGs in circulating immune cells in young animals than in old animals. While the number of Treg cells and the ratio of Treg among all T cells increases in both young and old mice with exercise, exercise is associated with a shift toward anti-inflammatory monocytes only in young animals.

In summary, our single-cell atlas, in combination with the single myofiber transcriptomic profiles, provides a rich source to study the changes in the cell type composition, transcriptional network, and communication patterns during aging and with physical exercise. Elucidating the mechanisms by which exercise modulates these intercellular and intracellular processes has great 
bioRxiv preprint doi: https://doi.org/10.1101/2022.01.12.475145; this version posted January 12, 2022. The copyright holder for this preprint (which was not certified by peer review) is the author/funder. All rights reserved. No reuse allowed without permission.

potential for the discovery of molecules and pathways that can be targeted therapeutically to reduce age-related diseases and to increase organismal health span. 


\section{AUTHOR CONTRIBUTIONS}

L.L. and T.A.R. conceived of the study and designed the experiments. L.L., M.T.B., J.M.R., S.K., A.L., M.M., C.M-R, H.I and M.J. carried out the experiments. L.L., M.T.B., J.M.R., S.K., L.T. and M.W. performed data analysis. J.C.W., M.A.G., A. B. and T.A.R. provided supervision and guidance. All authors participated in data interpretation. L.L. and T.A.R. wrote the manuscript with the input of all of the coauthors.

\section{ACKNOWLEDGEMENTS}

We thank all members of the Rando, Brunet and Goodell Laboratory for helpful discussions. We thank Brandon Carter for assistance at PAVIR FACS core, and Stanford Genome Technology Center for assistance in high-throughput sequencing. This work was supported by the Glenn Foundation for Medical Research, and by grants from the Department of Veterans Affairs (BLR\&D and RR\&D Merit Reviews) and the NIH (P01 AG036695, R37 AG023806, R01 AR073248, and TR01 AG047820) to T.A.R. 


\section{FIGURE LEGENDS}

Figure 1: Changes in cellular composition in stem cells in three tissues and their respective niches in response to aging and exercise revealed by scRNA-seq.

(A) Schematic diagram of the multi-tissue scRNA-seq methodology (n=4/group). (B) UMAP of the multi-tissue exercise single cell atlas. Cell clusters are colored by the tissue type. The total number of cells in each tissue is indicated in the legend. BL: blood; BM: bone marrow; MU: muscle; SVZ: subventricular zone; ECs: endothelial cells; FAPs: fibro-adipogenic progenitors; HSCs: hematopoietic stem cells; OPCs: oligodendrocyte progenitor cells; MuSCs: muscle stem cells; aNSCs: activated neural stem cells; qNSCs: quiescent neural stem cells; SMCs: smooth muscle cells. (C) UMAP of cell types in the SVZ. Fraction of each cell type is shown on the right. (D) Heatmap of cell density of the qNSCs, aNSCs and neuroblasts in control or exercised young and old mice. Scale bars represent cell density. (E) UMAP of the HSCs. Fraction of each cell type is shown on the right. MyMPP: myeloid multipotent progenitor; LMPP: lymphoidprimed multipotent progenitors; LyMPP: lymphoid multipotent progenitor; LTHSC: long-term hematopoietic stem cell; MEP: Megakaryocyte/erythroid progenitor; CDP: Common dendritic cell progenitor. (F) Heatmap of cell density of the HSC compartment in control or exercised young and old mice. (G) UMAP of subclusters in MuSCs. Note that cluster 1 and 3 diminished with age but only cluster 1 was restored after exercise. (H) Ridge plots of the expression of myogenic markers Pax7 and Myod1 in the MuSCs clusters. (I) Heatmap of markers genes for the MuSCs clusters. 


\section{Figure 2: Effect of aging on the transcriptome of stem cells and niches cells.}

(A) Summary of the number of upregulated and downregulated age-DEGs in major cell clusters in all 3 tissues. (B) Venn diagrams demonstrating the percentage of common age-DEGs in the common cell types from different tissues. (C) Dot plot summarizing the top 10 (ranked by P value) biological pathways enriched among age-DEGs in endothelial cells of the muscle (MU) and the SVZ. (D) Upset plot demonstrating common upregulated age-DEGs among major cell types in the SVZ. Oligo: oligodendrocytes, blasts: neuroblasts. (E) Table listing cell types in which members of the Hsp90 family members were downregulated. (F) Dot plot summarizing common biological pathways enriched among upregulated age-DEGs in major cell types of muscle, the SVZ and hematopoietic system. (G) Dot plot summarizing common biological pathways enriched among downregulated age-DEGs in major cell types of muscle, the SVZ and hematopoietic system.

\section{Figure 3: Reversal of age-induced gene expression changes by exercise.}

(A) Dot plot summarizing the number of Ex-DEGs in major cell types of the muscle, SVZ and hematopoietic system. (B) Bar graph demonstrating the percentage of exercise-restored genes in total age-DEGs in major cell types. (C) Dot plot summarizing immune pathways that are enriched in the downregulated Ex-DEGs in blood monocytes from young animals. (D) Dot plot summarizing the biological pathways enriched among exercise-restored genes.

(E) Chord plot demonstrating exercise-restored genes in various cell types that have implication in TNF $\alpha$ signaling. (F) Summary of differential inflammatory score in various cell types from young and old mice. 


\section{Figure 4: Cell-cell communication in the MuSC niche.}

(A) Dot plot summarizing the expression pattern of secreted and cell surface signaling molecules and their receptors in skeletal muscle. (B) Dot plot summarizing the expression pattern of ECM signaling molecules and their receptors in skeletal muscle. (C) Circle plot showing the aggregated cell-cell communication network mediated by secreted, cell-surface and ECM signaling molecules in the muscle of young mice. The thickness of the lines is determined by the overall signaling strength between the signal sending and receiving cell regardless of the direction. The size of the circles reflects the summarized signaling strength of a particular cell type. (D) Scatter plot showing the contribution of each cell type in the muscle in ECM-mediated communication. (E) Scatter plot showing the contribution of each cell type in the muscle in secreted/cell surface molecule-mediated communication. (F) Dot plot summarizing the signaling changes MuSCs receive from niche cells during aging. (G) Violin plots showing the expression pattern of Axl, Gas6 and Pros1 in muscle. (H) Violin plots showing the expression of $111 b$ and Osm in muscle monocytes. (I) Violin plots showing the expression of Fprl, Fpr2, Itgb2l and Itgam in muscle monocytes. (J) Chord plots showing cell-cell communication mediated by ANNEXIN, OSM and IL1 and THY1 in muscle. Note that ANNEXIN and OSM signaling disappears in O-C and recovers in O-Ex.

\section{Figure 5: Cell-cell communication in the SVZ NSC niche.}

(A) Dot plot summarizing the expression pattern of secreted and cell surface signaling molecules and their receptors in the SVZ. (B) Dot plot summarizing the expression pattern of ECM signaling molecules and their receptors in the SVZ. (C) Circle plot showing the aggregated cellcell communication network mediated by secreted, cell-surface and ECM signaling molecules in 
the SVZ of young mice. (D) Scatter plot showing the contribution of each cell type in the SVZ in ECM-mediated communication. (E) Scatter plot showing the contribution of each cell type in the SVZ in secreted/cell surface molecule-mediated communication. (F) Heatmaps showing the differential overall signaling strength in the SVZ between young and old mice (left) and between old mice without and with exercise (right). The top bars and right bars represent the sum of incoming and outgoing signaling strength of each cell type, respectively. In the scale bar, red and blue represent increased and decreased signaling in the second dataset, respectively. (G) Chord plots showing the TWEAK and MK signaling network in the SVZ. Note that TWEAK signaling disappears in O-C and recovers in O-Ex. (H) Circle plot demonstrating the TWEAK signaling network in the SVZ. The thickness of the lines represents signaling strength; the color of the lines represents the source of the signal and the arrows point toward signal receiving cells. (I) Violin plots showing the expression pattern of the TNFSF12 ligand and the TNFRSF12A receptor in the SVZ. (J) Chord plots showing the CSF, BAFF, TNF, THY1 AND CX3C signaling network in the SVZ. (K) Violin plots showing the expression pattern of the BAFF, TNF and THY1 signaling components in microglia.

Figure 6: Aging and exercise-induced changes in immune cells.

(A) Dot plot showing the percentage of T cell subtypes. (B) UMAP showing Ly6c2 expression in monocytes from the peripheral blood and the percentage of Ly6c $2^{+}$and Ly6c2- monocytes in YC, Y-Ex, O-C and O-Ex mice. (C) Dot plot summarizing the expression pattern of secreted and cell surface signaling molecules and their receptors in immune cells in the peripheral blood. (D) Circle plot demonstrating the CD39 signaling network in peripheral blood cells. The thickness of the lines represents signaling strength; the color of the lines represents the source of the signal 
and the arrows point toward signal receiving cells. (E) Violin plots showing the expression of Entpd1 (encoding CD39) and receptor Adora2a in immune cells. (F) Chord plots showing the GALECTIN signaling network in peripheral blood immune cells. (G) Heatmap showing the outgoing and incoming signaling pathways in Ly6c2+ and Ly6c2- monocytes in Y-C and Y-Ex mice. (H) Violin plot showing the expression Pf4 in peripheral blood immune cells.

\section{Figure 7: Aging and exercise-induced changes in myofibers.}

(A) Schematic diagram of the single fiber RNAseq methodology (top) and PCA analysis of fibers from young and old mice before and after exercise (bottom). (B) Venn diagram demonstrating the percentage of downregulated age-DEGs that were restored in myofibers from O-Ex mice (top) and dot plot summarizing the changes in the expression of genes in the lipid metabolic process (bottom). (C) Venn diagram demonstrating the percentage of upregulated ageDEGs that were restored in myofibers from O-Ex mice (top) and dot plot summarizing the changes in the expression of genes in the TNF $\alpha$ signaling pathway (bottom). (D) Dot plot demonstrating age-DEGs encoding secreted or cell surface ligand in myofibers (left) and the change in their expression in O-Ex mice (right). The color indicates fold change and the size indicates the significance of the change. (E) Upset plot showing the predicted receptors for Spp1 in cell types in skeletal muscle. (F) Dot plot demonstrating the number of percentage of predicted SPP1 target genes whose expression was restored in exercised old mice in muscle cell types. (G) Dot plot summarizing the biological pathways enriched among exercise-restored SPP1 target genes. 


\section{SUPPLEMENTAL INFORMATION}

\section{Materials and Methods}

\section{Animals and Treatments}

Male C57BL/6J mice of 4- and 22-months of age were acquired from NIA and housed in the Veterinary Medical Unit at the Veterans Affairs Palo Alto Health Care System. Animal protocols were approved by the Institutional Animal Care and Use Committee. For voluntary wheel running, mice were housed individually for 5 weeks in polycarbonate cages with $12.7-\mathrm{cm}$ diameter wheels equipped with optical rotation sensors (Lafayette Instrument, 80820). Nonexercised control mice were housed in identical cages with the wheels removed.

\section{Cell Isolation}

Cardiac puncture was performed to collect blood from anesthetized mice using syringes and tubes treated to prevent coagulation. The blood was diluted with equal volume of Dulbecco's phosphate-buffered saline (PBS) and overlaid onto Lymphoprep (Stem Cell Technologies). White blood cells were collected after gradient centrifugation. Live cells were sorted in the presence of DAPI for single cell capture. SVZ neurogenic niches were collected and processed as previously described (Dulken et al., 2019). Briefly, after blood collection, mice were perfused with $15 \mathrm{ml}$ of PBS with heparin sodium salt (50 U/ml) (Sigma-Aldrich, H3149-50KU) to remove residue blood. Microdissection of the SVZ was performed immediately following perfusion. The dissociated cells from the SVZ were then centrifuged through 22\% Percoll (Sigma-Aldrich, GE17-0891-01) in PBS to remove myelin debris. After centrifugation, cells were filtered through 
a 35- $\mu$ m snapcap filter (Corning, 352235), washed once with $1.5 \mathrm{ml}$ of FACS buffer (HBSS (Thermo-Fisher, 14175103), 1\% bovine serum albumin (Sigma, A7979), 0.1\% glucose (SigmaAldrich, G7021) and spun down for 5 min at 300g. Cells were then resuspended in FACS buffer with $1 \mu \mathrm{g} / \mathrm{ml}$ propidium iodide (PI) (BioLegend, 421301). FACS sorting was performed on a BD FACS Aria II sorter using a 100- $\mu$ m nozzle. Isolation of cells from skeletal muscle by sequential enzymatic and physical dissociation was performed as previously described (Liu et al., 2015). Briefly, hindlimb muscles were incubated with collagenase II and dispase sequentially before physical trituration. The resulting cell suspension was filtered through $40 \mu \mathrm{m}$ cell sieges to remove myofiber debris. Cells were then sorted in the presence of DAPI using a $70 \mu \mathrm{m}$ nozzle on a BD FACS Aria II sorter and live cells were collected for single cell capture. HSCs were enriched from the bone marrow as previous described (Challen et al., 2009). Briefly, bone marrow was isolated from femurs, tibiae and iliac crests. Side population staining was performed with Hoechst 33342 (Sigma). Magnetic enrichment was carried out on an AutoMACS using microbeads conjugated to antibodies recognizing biotin or mouse CD117 (all from Miltenyi Biotec). HSCs $\left(\mathrm{CD} 45.2^{+}\right.$, side population $\left.{ }^{\mathrm{KLS}}, \mathrm{CD} 150^{+}\right)$were sorted in the presence of $1 \mu \mathrm{g} / \mathrm{ml} \mathrm{PI}$ with a $70 \mu \mathrm{m}$ nozzle on a BD FACS Aria 3.3 sorter.

\section{Single cell RNA sequencing}

Single cells were captured in droplet emulsions using a 10x Chromium Controller (10x Genomics) with a target output of 10,000 cells per sample. scRNA-seq libraries were constructed as per 10x Genomics protocol using GenCode Single-Cell 3' Gel Bead and Library V3 kit according to manufacturer's instructions. The libraries were then pooled and sequenced on a NovaSeq6000 Sequencing System (Illumina) according to manufacturer's instructions. 


\section{scRNA-seq Data Processing}

Sequences were demultiplexed using CellRanger version 2.0 (10x Genomics) with default parameters. Reads were aligned to the mm10plus genome using STAR. Gene counts were produced using HTSEQ with default parameters except "stranded" was set to false and "mode" was set to intersection-nonempty. Only cells with >500 genes, $>1000$ UMIs, and $<10 \%$ mitochondrial reads were included in our analysis. Scaling, normalization, variable gene selection, dimensionality reduction, and clustering were performed with default settings using the Seurat package version 3.1 (Stuart et al., 2019). Cell types were assigned to each cluster using known marker genes. Clusters of doublets were identified and removed by careful manual inspection. DEGs were identified using the FindAllMarkers command in the Seurat package.

\section{Intercellular Interaction Analysis}

Intercellular interaction analysis was performed using the CellPhoneDB (Efremova et al., 2020) and CellChat (Jin et al., 2021) packages with standard parameters. Receptors and target genes for selected pathways were predicted using the NicheNet package (Browaeys et al., 2020).

\section{Single Fiber Isolation}

Single muscle fibers were prepared essentially as described (Rosenblatt et al., 1995). Briefly, the extensor digitorum longus (EDL) muscles were carefully dissected from mice following euthanasia and digested in $2 \mathrm{mg} / \mathrm{ml}$ collagenase II prepared in Ham's F10 with 10\% horse serum at $37^{\circ} \mathrm{C}$ for $80 \mathrm{~min}$ with gentle agitation. The digested EDL muscles were then triturated with a wide bore glass pipet in $20 \mathrm{ml} 10 \%$ horse serum in Ham's F10 in a $10 \mathrm{~cm}$ tissue culture dish. 
Individual fibers were washed 3 times in medium and incubated with $0.25 \%$ trypsin for 15 minutes. Trypsinized fibers were then rinse 3 times and centrifuged at $500 g$ for 5 minutes. Fibers were then snap-frozen in liquid nitrogen and stored at $-80^{\circ} \mathrm{C}$ until RNA extraction.

\section{RNA-seq}

RNA was extracted from fibers using the RNeasy Micro Plus Kit (Qiagen) according to manufacturer's instructions. Reverse transcription was performed with $10 \mathrm{ng}$ RNA using the SMART-Seq v4 Ultra Low Input RNA Kit (Takara) according to manufacturer's instructions. The cDNA was then sheared with a Covaris ultrasonicator and library constructions were performed with the Ovation Ultralow Multiplex system (NuGEN). Libraries underwent pairedend 101-bp sequencing on a HiSeq 4000 sequencer (Illumina) at the Stanford Genome Sequencing Service Center to a depth of 20-40 million reads. Reads were adapter- and qualitytrimmed with trim_galore (http://www.bioinformatics.babraham.ac.uk/projects/trim_galore) prior to mapping to the mouse genome assembly with STAR (Dobin et al., 2013). The number of reads in each gene was summarized using the package FeatureCounts (Liao et al., 2014) and DEGs were detected using the DESeq2 R package (Love et al., 2014). Gene Set Enrichment Analysis (Subramanian et al., 2005) was performed using the MSigDB Hallmark gene set and KEGG gene set. 


\section{Supplemental Figure Legends}

Figure S1: Construction of aging and exercise mouse single-cell atlases.

(A) Dot plot showing markers used for cell type annotation in the SVZ. (B) Dot plot showing markers used for cell type annotation in skeletal muscle. (C) Dot plot showing markers used for cell type annotation of the HSC compartment. (D) Dot plot showing markers used for cell type annotation in blood immune cells. (E) UMAP showing Louvain clusters of all cells under 4 different experimental conditions. (F) UMAP of cell types in skeletal muscle. Fraction of each cell type is shown on the right. (G) UMAP of cell types in peripheral blood. Fraction of each cell type is shown on the right.

Figure S2: Effect of aging on the transcriptome of stem cells and niche cells.

(A) Upset plot demonstrating common upregulated age-DEGs among major cell types in the hematopoietic system. (B) Upset plot demonstrating common downregulated age-DEGs among major cell types in the hematopoietic system. (C) List of upregulated age-DEGs among major cell types in the SVZ. (D) Dot plot demonstrating the expression of genes encoding enzymes involved in oxidative phosphorylation in SVZ cells from young and old animals. (E) Upset plot demonstrating common downregulated age-DEGs among major non-immune cell types in the SVZ.

Figure S3: Reversal of age-induced gene expression changes by exercise.

(A) Venn diagrams demonstrating the percentage of common Ex-DEGs in young and old cells.

(B) Violin plots showing the inflammatory scores of cells from the muscle. (C) Violin plots 
showing the inflammatory scores of cells from the SVZ. (D) Violin plots showing the inflammatory scores of hematopoietic cells.

\section{Figure S4: Cell-cell communication in the MuSC niche.}

(A) Circle plots showing the NOTCH, FGF, OSM and CXCL signaling networks in the muscle of young mice. The arrows point to the cell types that express the receptors. The colors of the lines represent the source of the ligands. The thickness of the lines represents signaling strength between the signal sending and receiving cell. (B) Heatmaps showing the differential overall signaling strength between young and old mice (left) and between old mice without and with exercise (right). The top bars and right bars represent the sum of incoming and outgoing signaling strength of each cell type, respectively. In the scale bar, red and blue represent increased and decreased signaling in the first dataset, respectively. (C) Heatmaps showing the outgoing and incoming signaling pathways in various cell types in the skeletal muscle that change with age in young (Y-C) and old (O-C) mice and old mice after exercise (O-Ex). The pathways are ranked by the differential overall signaling flow between the $\mathrm{Y}-\mathrm{C}$ and $\mathrm{O}-\mathrm{C}$ conditions. The top colored bars represent the overall signaling strength in each cell type. The horizontal gray bars represent the summarized strength of each signaling pathway from all cell types in the muscle. The pathways that were restored by exercise in old mice are printed in green. The pathways that were activated by exercise are printed in red.

\section{Figure S5: Cell-cell communication in the SVZ NSC niche.}

(A) Heatmaps showing the outgoing and incoming signaling patterns mediated by secreted and cell surface molecules in the SVZ. (B) Circle plot showing the WNT and EPHA signaling 
networks in the muscle of young mice. (C) Heatmaps showing the outgoing and incoming signaling pathways in various cell types in the SVZ that change with age in young (Y-C) and old (O-C) mice and old mice after exercise (O-Ex). The pathways are ranked by the differential overall signaling flow between the $\mathrm{Y}-\mathrm{C}$ and $\mathrm{O}-\mathrm{C}$ conditions. The top colored bars represent the overall signaling strength in each cell type. The horizontal gray bars represent the summarized strength of each signaling pathway from all cell types in the muscle. The pathways that were restored by exercise in old mice are printed in green. The pathways that were activated by exercise are printed in red.

Figure S6: Aging and exercise-induced changes in immune cells.

(A) UMAP of cell types in peripheral blood. Fraction of each cell type is shown on the right. (B) Table summarizing percentage of each immune cell type in the blood. (C) Table summarizing the percentage of $\mathrm{T}$ cells subtypes in the total $\mathrm{T}$ cell pool. (D) Heatmaps showing the outgoing and incoming signaling pathways in various cell types in the SVZ that change with age in young (Y-C) and old (O-C) mice and old mice after exercise (O-Ex). The pathways are ranked by the differential overall signaling flow between the Y-C and O-C conditions. The top colored bars represent the overall signaling strength in each cell type. The horizontal gray bars represent the summarized strength of each signaling pathway from all cell types in the muscle. The pathways that were restored by exercise in old mice are labeled in green. The pathways that were activated by exercise are labeled in red.

Figure S7: Aging and exercise-induced changes in myofibers. 
(A) Dot plot summarizing biological pathways enriched in the two major principle components that distinguish fiber transcriptomes by age and exercise status. (B) Dot plot summarizing biological pathways enriched in exercised induced genes in muscle fibers from young (left) and old (right) animals. (C) Dot plot summarizing genes encoding secreted ligands that were induced by exercise in muscle fibers from young (left) and old (right) animals. (D) Dumbbell plots demonstrating SPP1 target genes whose expression was reversed in muscle cell types in exercised old mice. The $\mathrm{x}$ axis represents scaled relative expression. 


\section{REFERENCES}

Bhaskar, K., Konerth, M., Kokiko-Cochran, O.N., Cardona, A., Ransohoff, R.M., and Lamb, B.T. (2010). Regulation of tau pathology by the microglial fractalkine receptor. Neuron 68, 1931.

Brett, J.O., Arjona, M., Ikeda, M., Quarta, M., de Morree, A., Egner, I.M., Perandini, L.A., Ishak, H.D., Goshayeshi, A., Benjamin, D.I., et al. (2020). Exercise rejuvenates quiescent skeletal muscle stem cells in old mice through restoration of Cyclin D1. Nat Metab 2, 307-317. Browaeys, R., Saelens, W., and Saeys, Y. (2020). NicheNet: modeling intercellular communication by linking ligands to target genes. Nat Methods 17, 159-162.

Brunet, A., and Rando, T.A. (2017). Interaction between epigenetic and metabolism in aging stem cells. Curr Opin Cell Biol 45, 1-7.

Challen, G.A., Boles, N., Lin, K.K., and Goodell, M.A. (2009). Mouse hematopoietic stem cell identification and analysis. Cytometry A 75, 14-24.

Chandel, N.S., Jasper, H., Ho, T.T., and Passegue, E. (2016). Metabolic regulation of stem cell function in tissue homeostasis and organismal ageing. Nat Cell Biol 18, 823-832.

Cho, S.H., Sun, B., Zhou, Y., Kauppinen, T.M., Halabisky, B., Wes, P., Ransohoff, R.M., and Gan, L. (2011). CX3CR1 protein signaling modulates microglial activation and protects against plaque-independent cognitive deficits in a mouse model of Alzheimer disease. J Biol Chem 286, 32713-32722.

Conboy, I.M., Conboy, M.J., Smythe, G.M., and Rando, T.A. (2003). Notch-mediated restoration of regenerative potential to aged muscle. Science 302, 1575-1577.

De Miguel, Z., Khoury, N., Betley, M.J., Lehallier, B., Willoughby, D., Olsson, N., Yang, A.C., Hahn, O., Lu, N., Vest, R.T., et al. (2021). Exercise plasma boosts memory and dampens brain inflammation via clusterin. Nature 600, 494-499.

Deaglio, S., Dwyer, K.M., Gao, W., Friedman, D., Usheva, A., Erat, A., Chen, J.F., Enjyoji, K., Linden, J., Oukka, M., et al. (2007). Adenosine generation catalyzed by CD39 and CD73 expressed on regulatory T cells mediates immune suppression. J Exp Med 204, 1257-1265. Dobin, A., Davis, C.A., Schlesinger, F., Drenkow, J., Zaleski, C., Jha, S., Batut, P., Chaisson, M., and Gingeras, T.R. (2013). STAR: ultrafast universal RNA-seq aligner. Bioinformatics 29, 15-21.

Dulken, B.W., Buckley, M.T., Navarro Negredo, P., Saligrama, N., Cayrol, R., Leeman, D.S., George, B.M., Boutet, S.C., Hebestreit, K., Pluvinage, J.V., et al. (2019). Single-cell analysis reveals $\mathrm{T}$ cell infiltration in old neurogenic niches. Nature 571, 205-210.

Efremova, M., Vento-Tormo, M., Teichmann, S.A., and Vento-Tormo, R. (2020). CellPhoneDB: inferring cell-cell communication from combined expression of multi-subunit ligand-receptor complexes. Nat Protoc 15, 1484-1506.

Fang, X., Han, D., Cheng, Q., Zhang, P., Zhao, C., Min, J., and Wang, F. (2018). Association of Levels of Physical Activity With Risk of Parkinson Disease: A Systematic Review and Metaanalysis. JAMA Netw Open 1, e182421.

Fontana, L., and Partridge, L. (2015). Promoting health and longevity through diet: from model organisms to humans. Cell 161, 106-118.

Gage, F.H., and Temple, S. (2013). Neural stem cells: generating and regenerating the brain.

Neuron 80, 588-601. 
Goodell, M.A., and Rando, T.A. (2015). Stem cells and healthy aging. Science 350, 1199-1204. Hambrecht, R., Adams, V., Erbs, S., Linke, A., Krankel, N., Shu, Y., Baither, Y., Gielen, S., Thiele, H., Gummert, J.F., et al. (2003). Regular physical activity improves endothelial function in patients with coronary artery disease by increasing phosphorylation of endothelial nitric oxide synthase. Circulation 107, 3152-3158.

Hernando-Herraez, I., Evano, B., Stubbs, T., Commere, P.H., Jan Bonder, M., Clark, S., Andrews, S., Tajbakhsh, S., and Reik, W. (2019). Ageing affects DNA methylation drift and transcriptional cell-to-cell variability in mouse muscle stem cells. Nat Commun 10, 4361. Horowitz, A.M., Fan, X., Bieri, G., Smith, L.K., Sanchez-Diaz, C.I., Schroer, A.B., Gontier, G., Casaletto, K.B., Kramer, J.H., Williams, K.E., et al. (2020). Blood factors transfer beneficial effects of exercise on neurogenesis and cognition to the aged brain. Science 369, 167-173.

Jin, S., Guerrero-Juarez, C.F., Zhang, L., Chang, I., Ramos, R., Kuan, C.H., Myung, P., Plikus, M.V., and Nie, Q. (2021). Inference and analysis of cell-cell communication using CellChat. Nat Commun 12, 1088.

Jones, D.L., and Rando, T.A. (2011). Emerging models and paradigms for stem cell ageing. Nat Cell Biol 13, 506-512.

Kraus, W.E., Houmard, J.A., Duscha, B.D., Knetzger, K.J., Wharton, M.B., McCartney, J.S., Bales, C.W., Henes, S., Samsa, G.P., Otvos, J.D., et al. (2002). Effects of the amount and intensity of exercise on plasma lipoproteins. N Engl J Med 347, 1483-1492.

Kummer, D., and Ebnet, K. (2018). Junctional Adhesion Molecules (JAMs): The JAM-Integrin Connection. Cells 7.

Liao, Y., Smyth, G.K., and Shi, W. (2014). featureCounts: an efficient general purpose program for assigning sequence reads to genomic features. Bioinformatics 30, 923-930.

Liu, L., Cheung, T.H., Charville, G.W., and Rando, T.A. (2015). Isolation of skeletal muscle stem cells by fluorescence-activated cell sorting. Nat Protoc 10, 1612-1624.

Liu, L., and Rando, T.A. (2011). Manifestations and mechanisms of stem cell aging. J Cell Biol 193, 257-266.

Love, M.I., Huber, W., and Anders, S. (2014). Moderated estimation of fold change and dispersion for RNA-seq data with DESeq2. Genome Biol 15, 550.

Lynch, C.N., Wang, Y.C., Lund, J.K., Chen, Y.W., Leal, J.A., and Wiley, S.R. (1999). TWEAK induces angiogenesis and proliferation of endothelial cells. J Biol Chem 274, 8455-8459.

Ma, S., Sun, S., Geng, L., Song, M., Wang, W., Ye, Y., Ji, Q., Zou, Z., Wang, S., He, X., et al. (2020). Caloric Restriction Reprograms the Single-Cell Transcriptional Landscape of Rattus Norvegicus Aging. Cell 180, 984-1001 e1022.

Mahmoudi, S., Mancini, E., Xu, L., Moore, A., Jahanbani, F., Hebestreit, K., Srinivasan, R., Li, X., Devarajan, K., Prelot, L., et al. (2019). Heterogeneity in old fibroblasts is linked to variability in reprogramming and wound healing. Nature 574, 553-558.

Manczak, M., Park, B.S., Jung, Y., and Reddy, P.H. (2004). Differential expression of oxidative phosphorylation genes in patients with Alzheimer's disease: implications for early mitochondrial dysfunction and oxidative damage. Neuromolecular Med 5, 147-162.

Martinez-Jimenez, C.P., Eling, N., Chen, H.C., Vallejos, C.A., Kolodziejczyk, A.A., Connor, F., Stojic, L., Rayner, T.F., Stubbington, M.J.T., Teichmann, S.A., et al. (2017). Aging increases cell-to-cell transcriptional variability upon immune stimulation. Science 355, 1433-1436.

Mizuno, T., Kawanokuchi, J., Numata, K., and Suzumura, A. (2003). Production and neuroprotective functions of fractalkine in the central nervous system. Brain Res 979, 65-70. 
Navarro Negredo, P., Yeo, R.W., and Brunet, A. (2020). Aging and Rejuvenation of Neural Stem Cells and Their Niches. Cell Stem Cell 27, 202-223.

Newman, A.M., Steen, C.B., Liu, C.L., Gentles, A.J., Chaudhuri, A.A., Scherer, F., Khodadoust, M.S., Esfahani, M.S., Luca, B.A., Steiner, D., et al. (2019). Determining cell type abundance and expression from bulk tissues with digital cytometry. Nat Biotechnol 37, 773-782.

Nieman, D.C., Johanssen, L.M., Lee, J.W., and Arabatzis, K. (1990). Infectious episodes in runners before and after the Los Angeles Marathon. J Sports Med Phys Fitness 30, 316-328. Nieman, D.C., and Wentz, L.M. (2019). The compelling link between physical activity and the body's defense system. J Sport Health Sci 8, 201-217.

Oike, Y., Akao, M., Kubota, Y., and Suda, T. (2005). Angiopoietin-like proteins: potential new targets for metabolic syndrome therapy. Trends Mol Med 11, 473-479.

Olingy, C.E., San Emeterio, C.L., Ogle, M.E., Krieger, J.R., Bruce, A.C., Pfau, D.D., Jordan, B.T., Peirce, S.M., and Botchwey, E.A. (2017). Non-classical monocytes are biased progenitors of wound healing macrophages during soft tissue injury. Sci Rep 7, 447.

Pawlikowski, B., Vogler, T.O., Gadek, K., and Olwin, B.B. (2017). Regulation of skeletal muscle stem cells by fibroblast growth factors. Dev Dyn 246, 359-367.

Pedersen, B.K., and Febbraio, M.A. (2012). Muscles, exercise and obesity: skeletal muscle as a secretory organ. Nat Rev Endocrinol 8, 457-465.

Perretti, M., and D'Acquisto, F. (2009). Annexin A1 and glucocorticoids as effectors of the resolution of inflammation. Nat Rev Immunol 9, 62-70.

Rando, T.A., and Wyss-Coray, T. (2014). Stem cells as vehicles for youthful regeneration of aged tissues. J Gerontol A Biol Sci Med Sci 69 Suppl 1, S39-42.

Ratajczak, M.Z., Majka, M., Kucia, M., Drukala, J., Pietrzkowski, Z., Peiper, S., and JanowskaWieczorek, A. (2003). Expression of functional CXCR4 by muscle satellite cells and secretion of SDF-1 by muscle-derived fibroblasts is associated with the presence of both muscle progenitors in bone marrow and hematopoietic stem/progenitor cells in muscles. Stem Cells 21, 363-371. Ren, R., Ocampo, A., Liu, G.H., and Izpisua Belmonte, J.C. (2017). Regulation of Stem Cell Aging by Metabolism and Epigenetics. Cell Metab 26, 460-474.

Rosenblatt, J.D., Lunt, A.I., Parry, D.J., and Partridge, T.A. (1995). Culturing satellite cells from living single muscle fiber explants. In Vitro Cell Dev Biol Anim 31, 773-779.

Rovio, S., Kareholt, I., Helkala, E.L., Viitanen, M., Winblad, B., Tuomilehto, J., Soininen, H., Nissinen, A., and Kivipelto, M. (2005). Leisure-time physical activity at midlife and the risk of dementia and Alzheimer's disease. Lancet Neurol 4, 705-711.

Rowe, G.C., Raghuram, S., Jang, C., Nagy, J.A., Patten, I.S., Goyal, A., Chan, M.C., Liu, L.X., Jiang, A., Spokes, K.C., et al. (2014). PGC-1alpha induces SPP1 to activate macrophages and orchestrate functional angiogenesis in skeletal muscle. Circ Res 115, 504-517.

Saint-Maurice, P.F., Coughlan, D., Kelly, S.P., Keadle, S.K., Cook, M.B., Carlson, S.A., Fulton, J.E., and Matthews, C.E. (2019). Association of Leisure-Time Physical Activity Across the Adult Life Course With All-Cause and Cause-Specific Mortality. JAMA Netw Open 2, e190355. Salzer, M.C., Lafzi, A., Berenguer-Llergo, A., Youssif, C., Castellanos, A., Solanas, G., Peixoto, F.O., Stephan-Otto Attolini, C., Prats, N., Aguilera, M., et al. (2018). Identity Noise and Adipogenic Traits Characterize Dermal Fibroblast Aging. Cell 175, 1575-1590 e1522. Scarmeas, N., Luchsinger, J.A., Schupf, N., Brickman, A.M., Cosentino, S., Tang, M.X., and Stern, Y. (2009). Physical activity, diet, and risk of Alzheimer disease. JAMA 302, 627-637. Schafer, M.K., and Frotscher, M. (2012). Role of L1CAM for axon sprouting and branching. Cell Tissue Res 349, 39-48. 
Seki, M., Oomizu, S., Sakata, K.M., Sakata, A., Arikawa, T., Watanabe, K., Ito, K., Takeshita, K., Niki, T., Saita, N., et al. (2008). Galectin-9 suppresses the generation of Th17, promotes the induction of regulatory $\mathrm{T}$ cells, and regulates experimental autoimmune arthritis. Clin Immunol $127,78-88$.

Shao, X., Liao, J., Li, C., Lu, X., Cheng, J., and Fan, X. (2021). CellTalkDB: a manually curated database of ligand-receptor interactions in humans and mice. Brief Bioinform 22.

Shen, B., Tasdogan, A., Ubellacker, J.M., Zhang, J., Nosyreva, E.D., Du, L., Murphy, M.M., Hu, S., Yi, Y., Kara, N., et al. (2021). A mechanosensitive peri-arteriolar niche for osteogenesis and lymphopoiesis. Nature 591, 438-444.

Sigal, R.J., Kenny, G.P., Boule, N.G., Wells, G.A., Prud'homme, D., Fortier, M., Reid, R.D., Tulloch, H., Coyle, D., Phillips, P., et al. (2007). Effects of aerobic training, resistance training, or both on glycemic control in type 2 diabetes: a randomized trial. Ann Intern Med 147, 357-369. Sorrelle, N., Dominguez, A.T.A., and Brekken, R.A. (2017). From top to bottom: midkine and pleiotrophin as emerging players in immune regulation. J Leukoc Biol 102, 277-286.

Stuart, T., Butler, A., Hoffman, P., Hafemeister, C., Papalexi, E., Mauck, W.M., 3rd, Hao, Y., Stoeckius, M., Smibert, P., and Satija, R. (2019). Comprehensive Integration of Single-Cell Data. Cell 177, 1888-1902 e1821.

Subramanian, A., Tamayo, P., Mootha, V.K., Mukherjee, S., Ebert, B.L., Gillette, M.A., Paulovich, A., Pomeroy, S.L., Golub, T.R., Lander, E.S., et al. (2005). Gene set enrichment analysis: a knowledge-based approach for interpreting genome-wide expression profiles. Proc Natl Acad Sci U S A 102, 15545-15550.

Tabula Muris, C. (2020). A single-cell transcriptomic atlas characterizes ageing tissues in the mouse. Nature 583, 590-595.

Tanaka, M., and Siemann, D.W. (2020). Gas6/Axl Signaling Pathway in the Tumor Immune Microenvironment. Cancers (Basel) 12.

Uysal, K.T., Wiesbrock, S.M., Marino, M.W., and Hotamisligil, G.S. (1997). Protection from obesity-induced insulin resistance in mice lacking TNF-alpha function. Nature 389, 610-614. van Praag, H., Christie, B.R., Sejnowski, T.J., and Gage, F.H. (1999). Running enhances neurogenesis, learning, and long-term potentiation in mice. Proc Natl Acad Sci U S A 96, 1342713431.

van Praag, H., Shubert, T., Zhao, C., and Gage, F.H. (2005). Exercise enhances learning and hippocampal neurogenesis in aged mice. J Neurosci 25, 8680-8685.

Wiley, S.R., Cassiano, L., Lofton, T., Davis-Smith, T., Winkles, J.A., Lindner, V., Liu, H., Daniel, T.O., Smith, C.A., and Fanslow, W.C. (2001). A novel TNF receptor family member binds TWEAK and is implicated in angiogenesis. Immunity 15, 837-846.

Xie, X.P., Laks, D.R., Sun, D., Poran, A., Laughney, A.M., Wang, Z., Sam, J., Belenguer, G., Farinas, I., Elemento, O., et al. (2020). High-resolution mouse subventricular zone stem-cell niche transcriptome reveals features of lineage, anatomy, and aging. Proc Natl Acad Sci U S A $117,31448-31458$.

Xu, H., Barnes, G.T., Yang, Q., Tan, G., Yang, D., Chou, C.J., Sole, J., Nichols, A., Ross, J.S., Tartaglia, L.A., et al. (2003). Chronic inflammation in fat plays a crucial role in the development of obesity-related insulin resistance. J Clin Invest 112, 1821-1830. 
Figure TioRxiv preprint doi: https://doi.org/10.1101/2022.01.12.475145; this version posted January 12,2022 . The copyright holder for this preprint
(which was not certified by peer review) is the author/funder. All rights reserved. No reuse allowed without permission.

Figure 1 ioRxiv preprint doi: https://doi.org/10.1101/2022.01.12.475145; this version posted January 12,2022 . The copyright holder for this preprint
(which was not certified by peer review) is the author/funder. All rights reserved. No reuse allowed without permission.

A

$\sigma^{\mathrm{C} 57 \mathrm{BI} / 6 \mathrm{~J} \text { mouse }}$



B



E
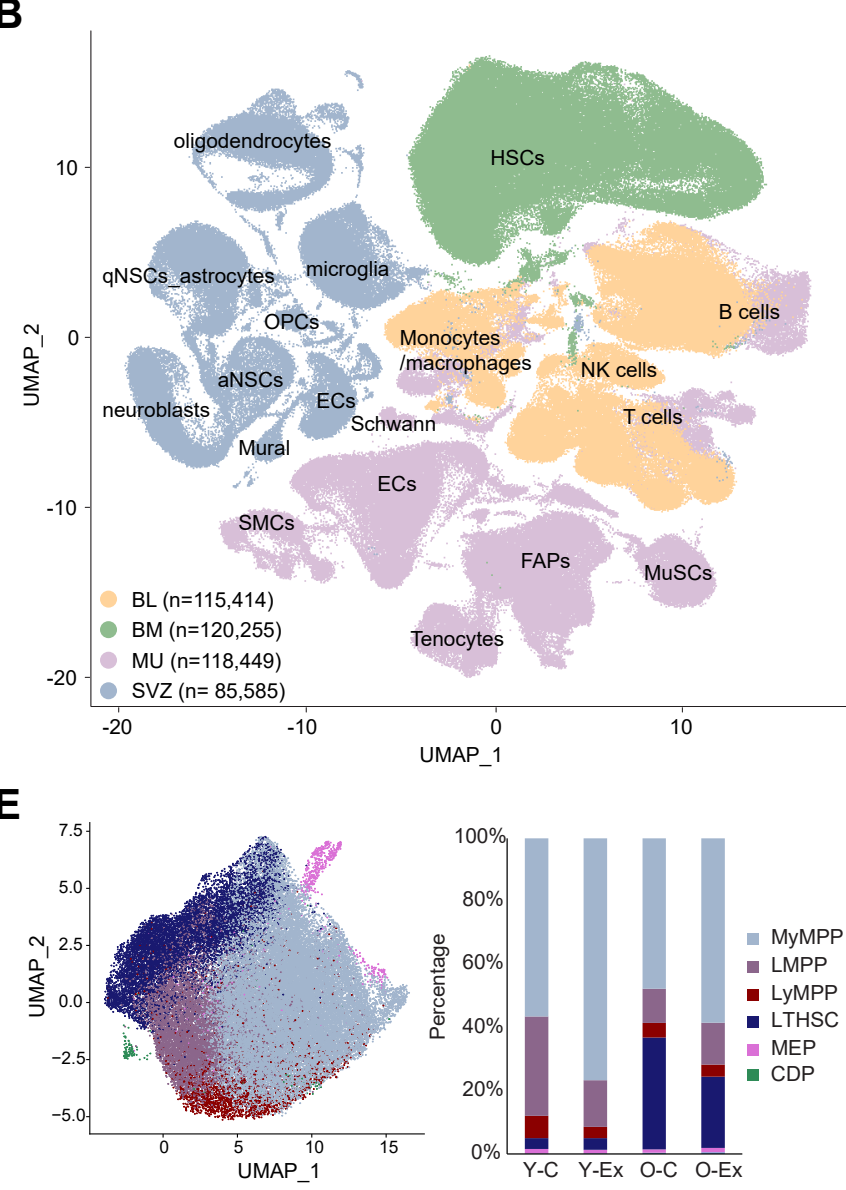

Y-C Y-Ex O-C O-Ex
C
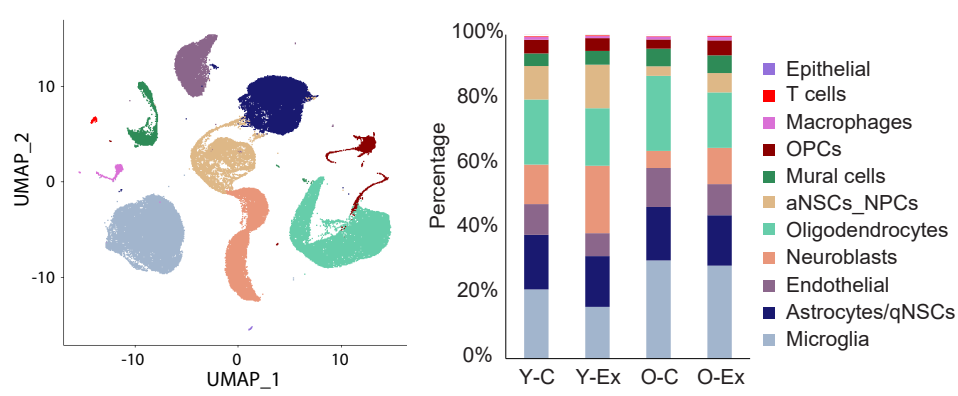

D



$\mathbf{F}$
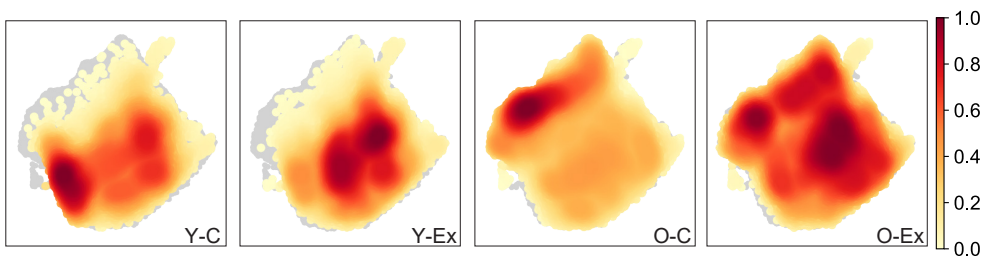

H
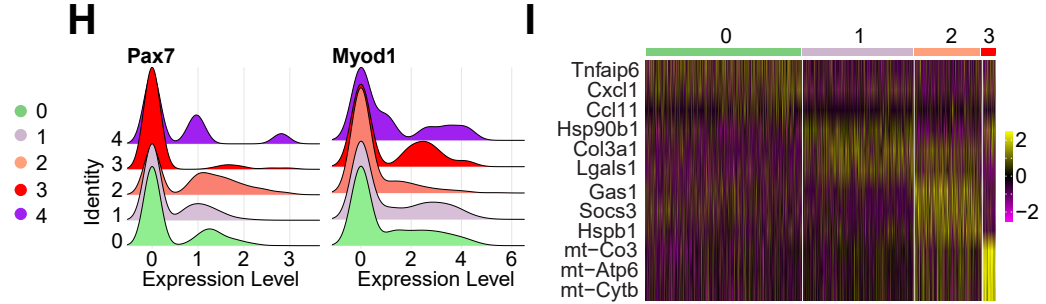

G

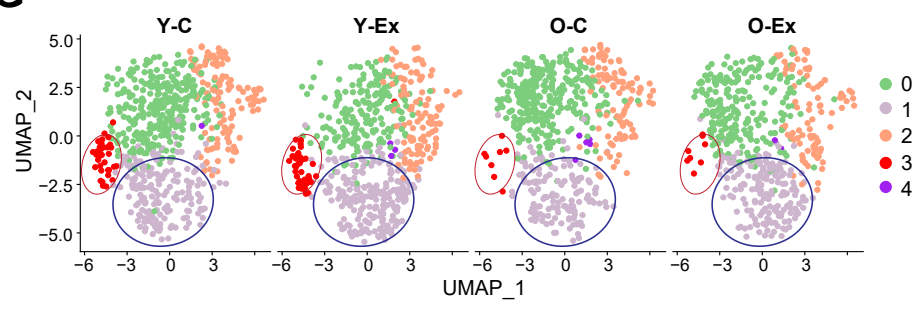

Aging and exercise atlas 435,628 cells

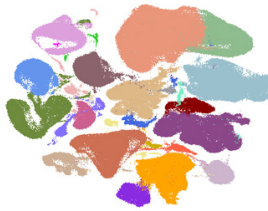

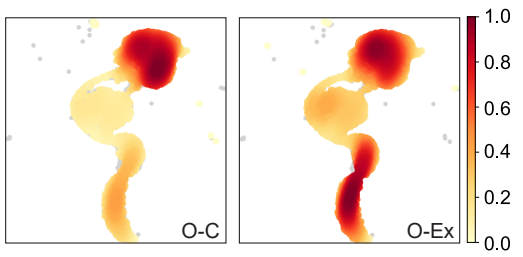

458,754 QC regression
cells

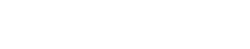

Epithelia

Oligodendrocytes

Astrocytes/qNSCs

(n)

1.0
0.8
0.6
0.4
0.2
0.0


Figure 2oRxiv preprint doi: https://doi.org/10.1101/2022.01.12.475145; this version posted January 12, 2022. The copyright holder for this preprint (which was not certified by peer review) is the author/funder. All rights reserved. No reuse allowed without permission.

A



B



C

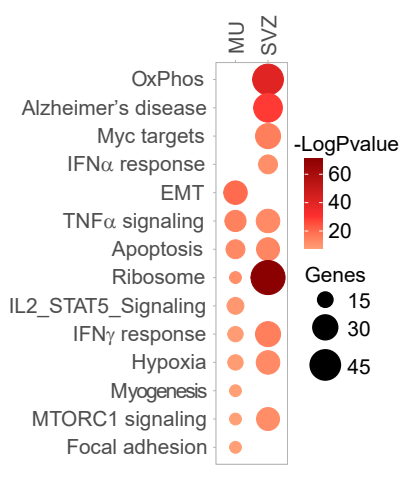

D

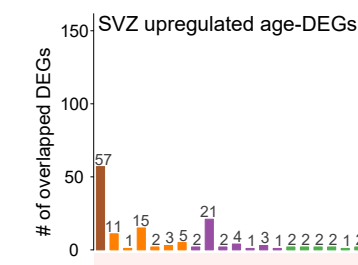

\author{
s
}
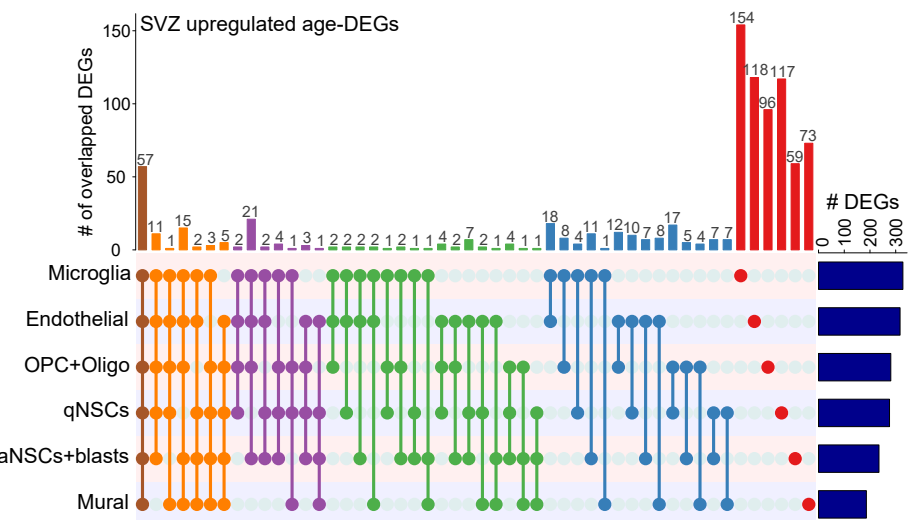

E

downregulated age-DEGs

$\mathbf{F}$

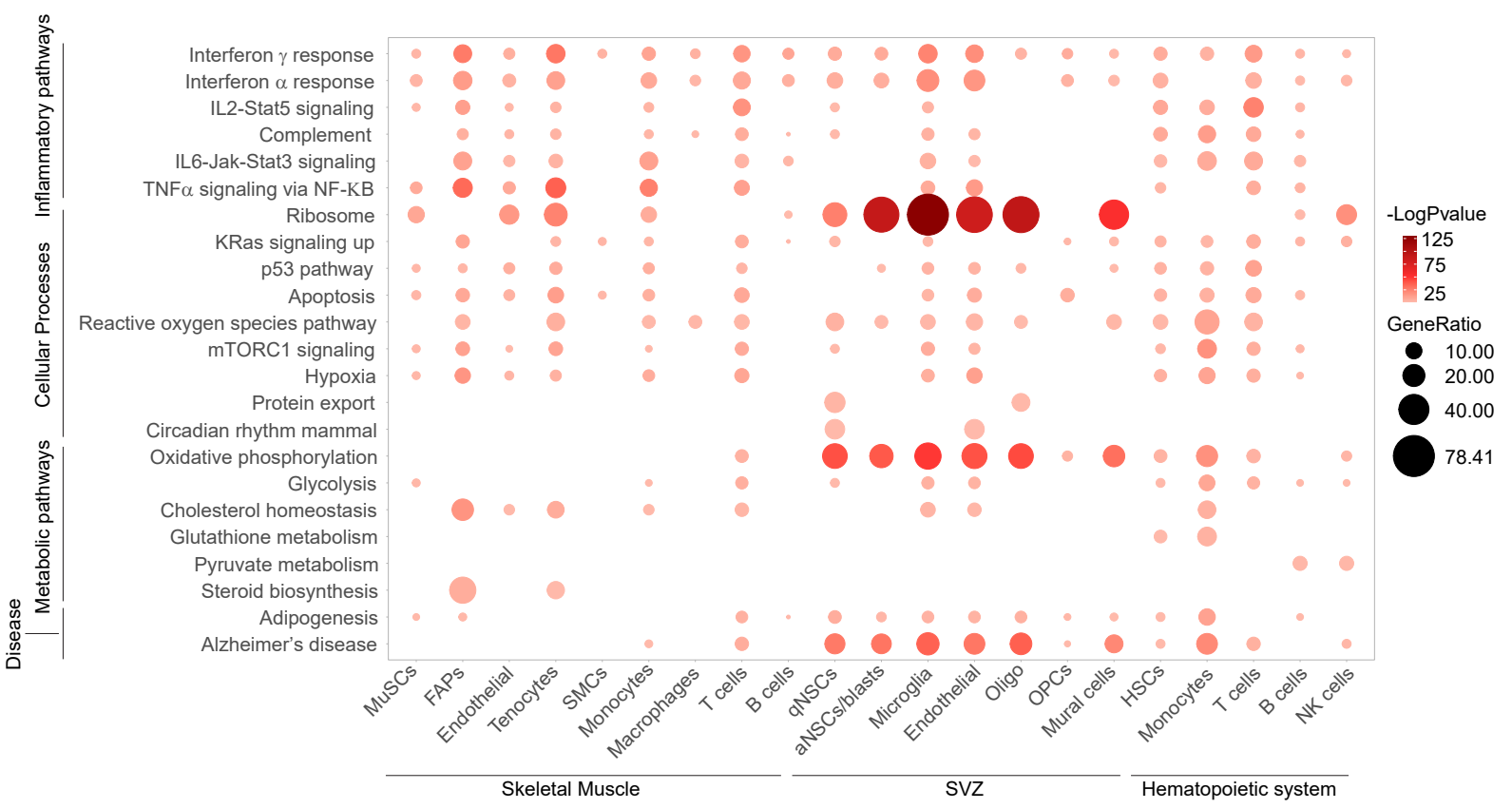

G




Figure BoRxiv preprint doi: https://doi.org/10.1101/2022.01.12.475145; this version posted January 12, 2022. The copyright holder for this preprint (which was not certified by peer review) is the author/funder. All rights reserved. No reuse allowed without permission.

A

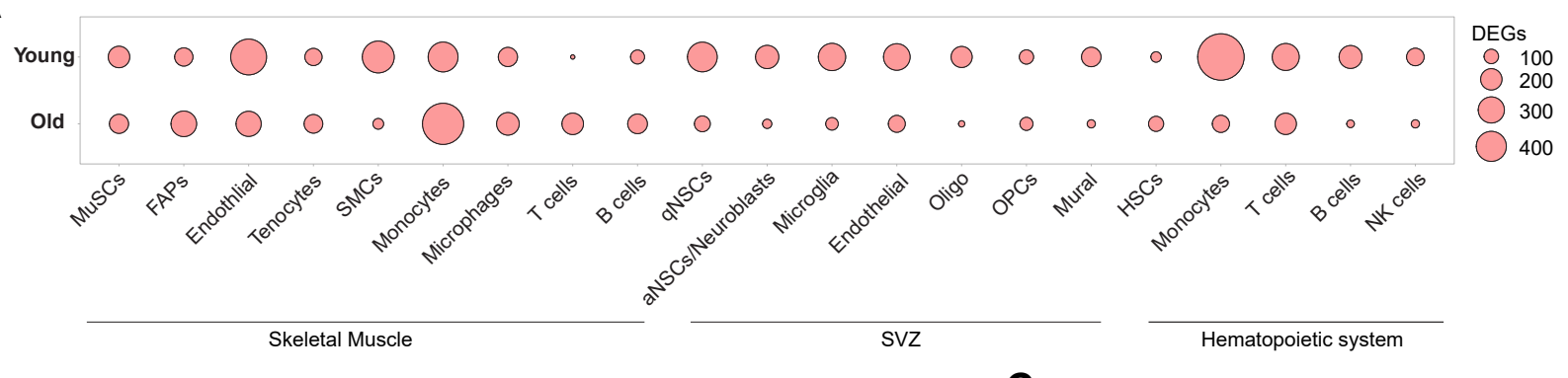

B

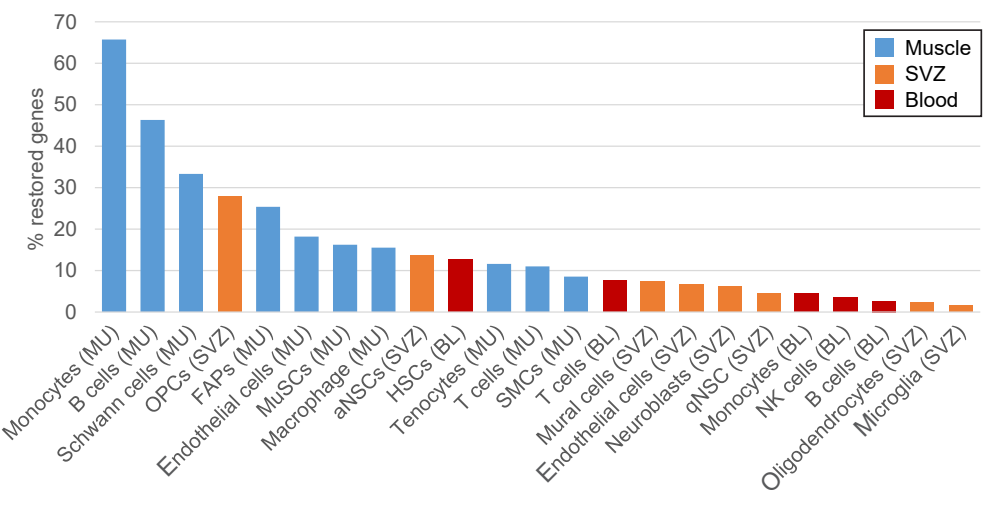

D
C

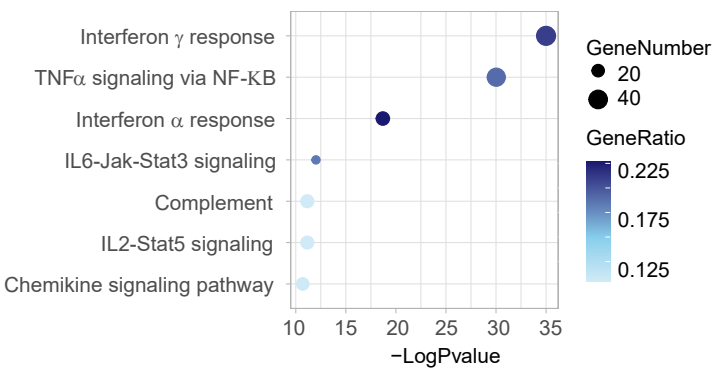




E

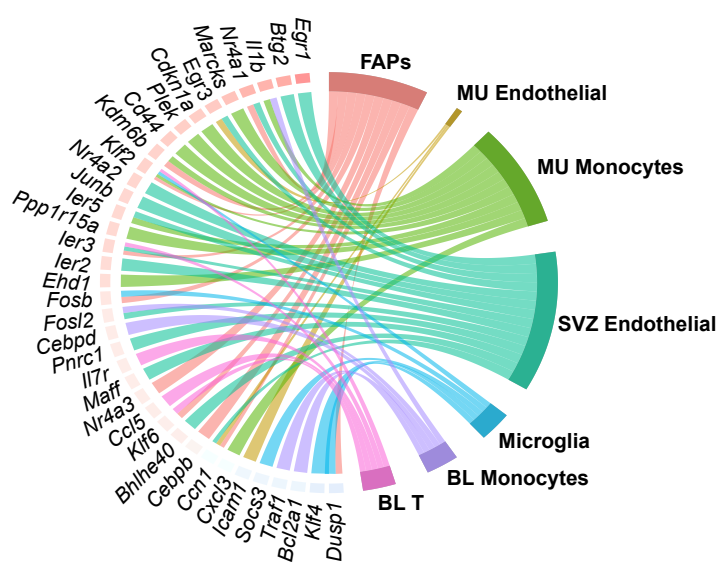



F

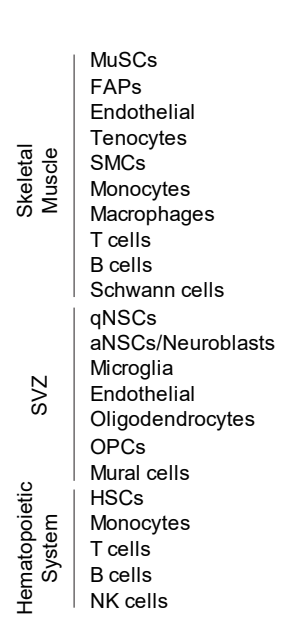




Figure 4oRxiv preprint doi: https://doi.org/10.1101/2022.01.12.475145; this version posted January 12 , 2022. The copyright holder for this preprint (which was not certified by peer review) is the author/funder. All rights reserved. No reuse allowed without permission.

A

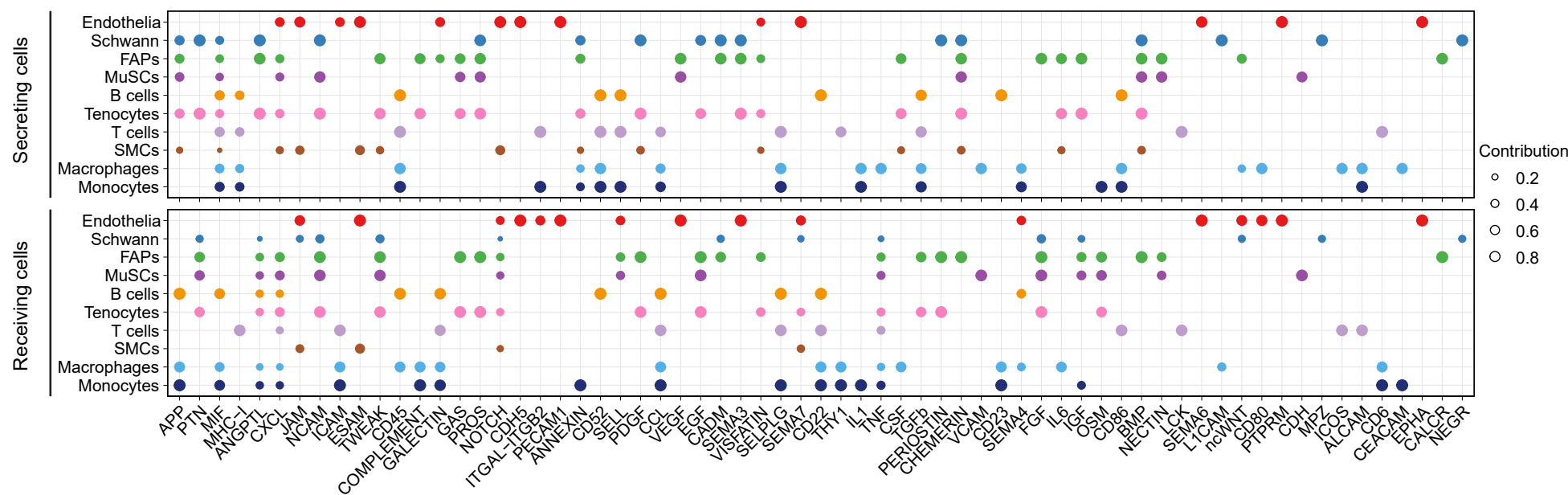

B

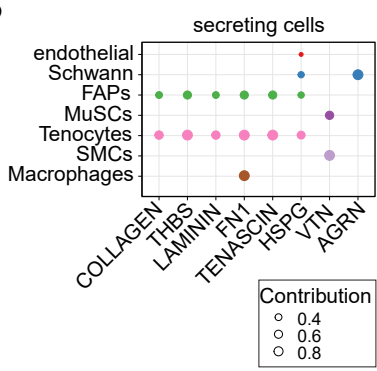

$\mathbf{F}$

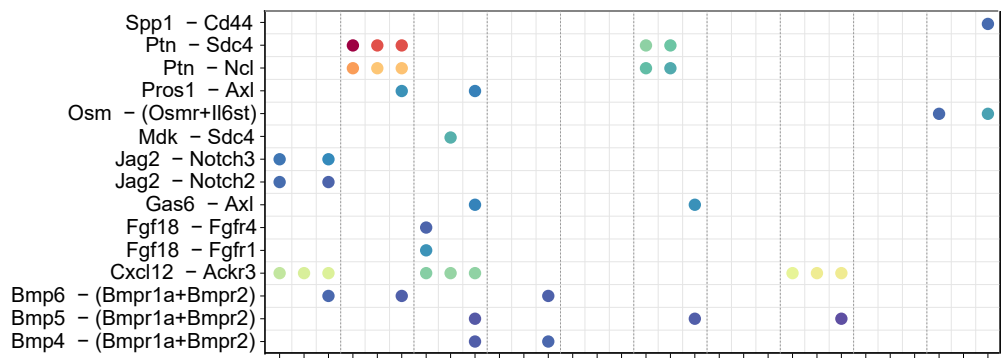



H
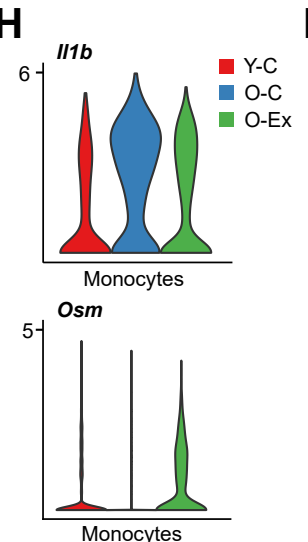

1
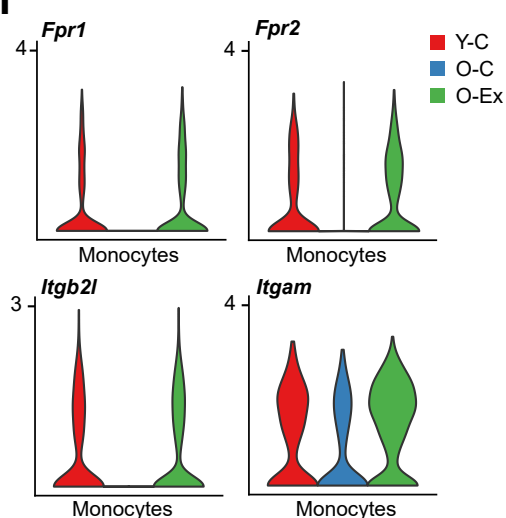

$\mathbf{J}$
C


G



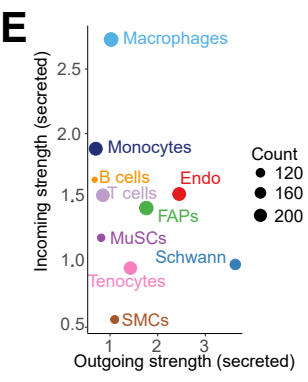

$\mathrm{Y}-\mathrm{C}$

O-C

O-Ex

- $p>0.05$

Commun. Prob.

$\max$

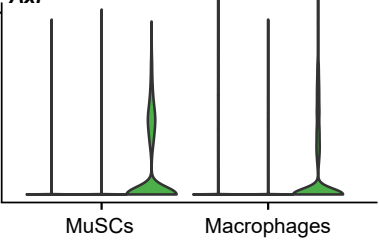

$\min$

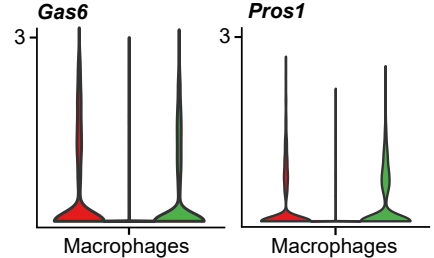

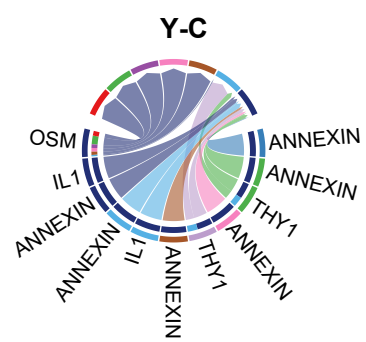

Endothelial T cells

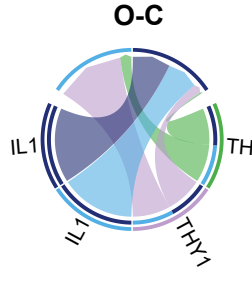

Schwann FAPs MuSCs SMCs

Macrophages

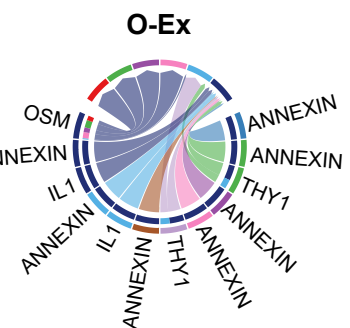

Tenocytes

Monocytes 
Figure 5oRxiv preprint doi: https://doi.org/10.1101/2022.01.12.475145; this version posted January 12 , 2022. The copyright holder for this preprint (which was not certified by peer review) is the author/funder. All rights reserved. No reuse allowed without permission.

A

B

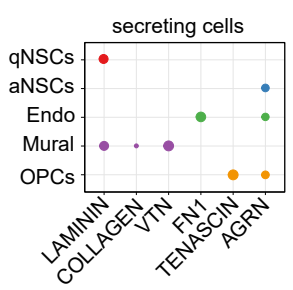

\begin{tabular}{lllll}
\hline Contribution & $\circ$ & 0.6 & $\circ$ & 0.9 \\
\hline
\end{tabular}

$\mathbf{F}$

$\mathrm{H}$



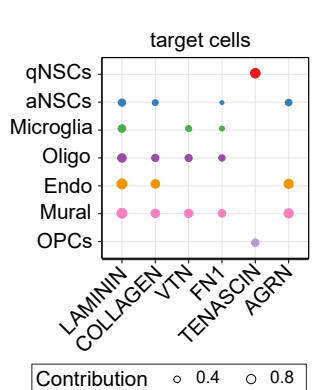

C

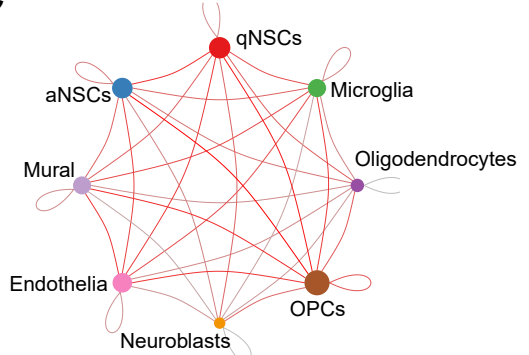

D

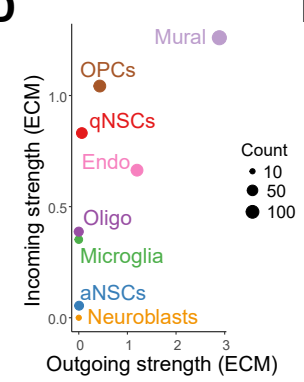

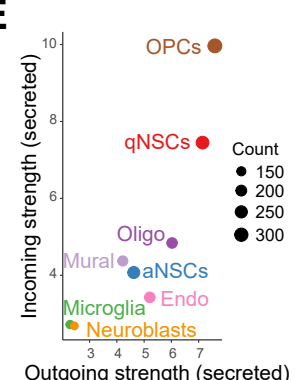
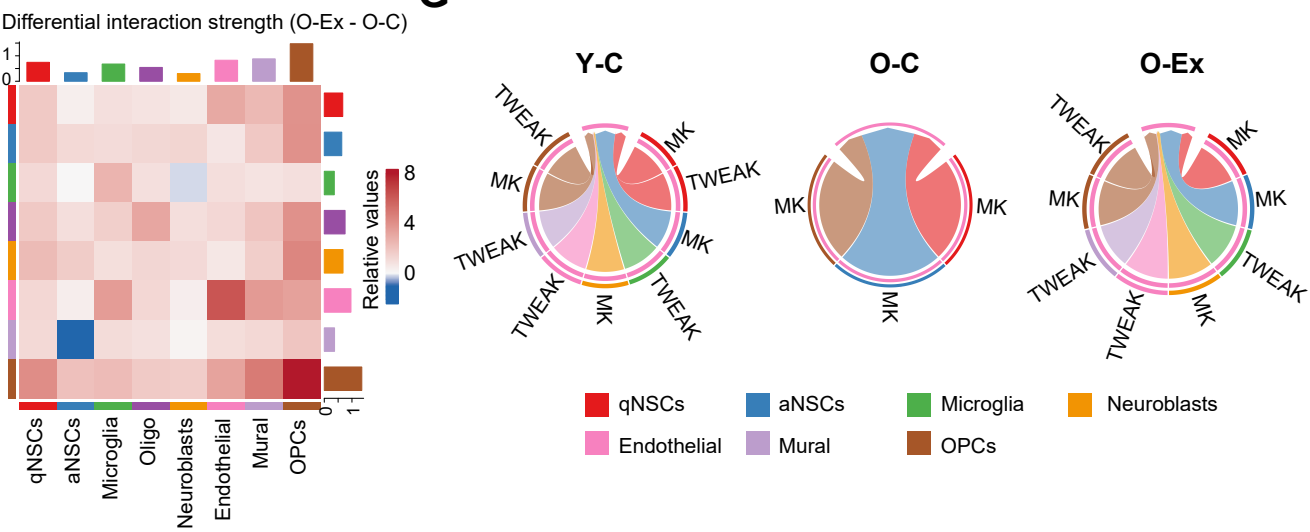

Endothelial Mural $\square$ OPCs

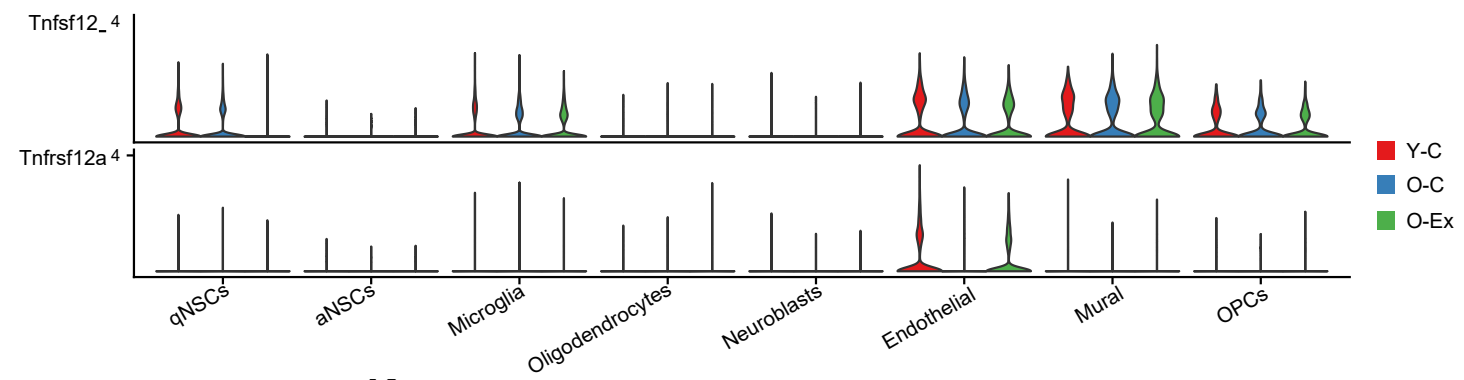

J


K
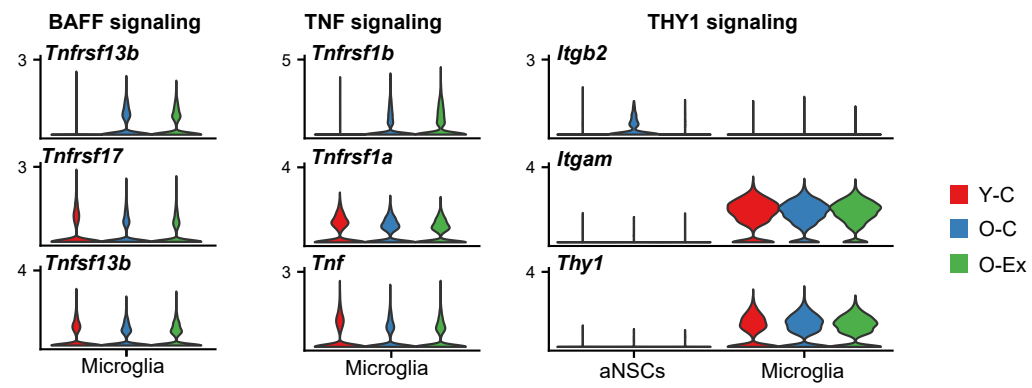

Microglia Mural Oligodendrocytes

Endothelial aNSCs $\square$ OPCs

$$
\frac{1}{\text { Microglia }}
$$



Figure GoRxiv preprint doi: https://doi.org/10.1101/2022.01.12.475145; this version posted January 12,2022 . The copyright holder for this preprint
(which was not certified by peer review) is the author/funder. All rights reserved. No reuse allowed without permission.

A

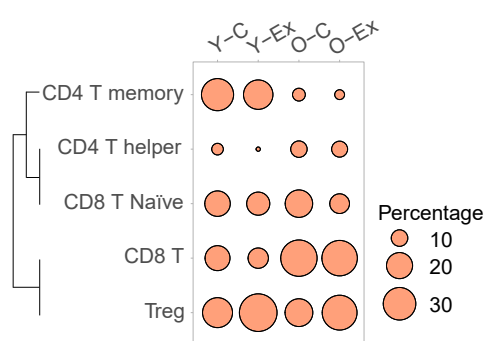

B
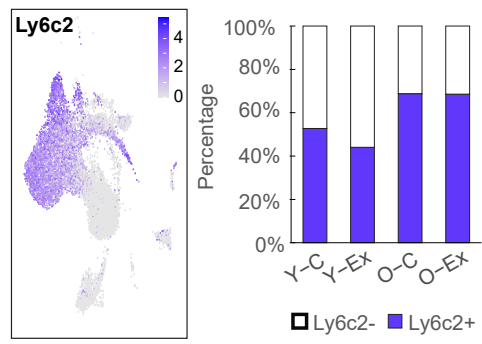

C

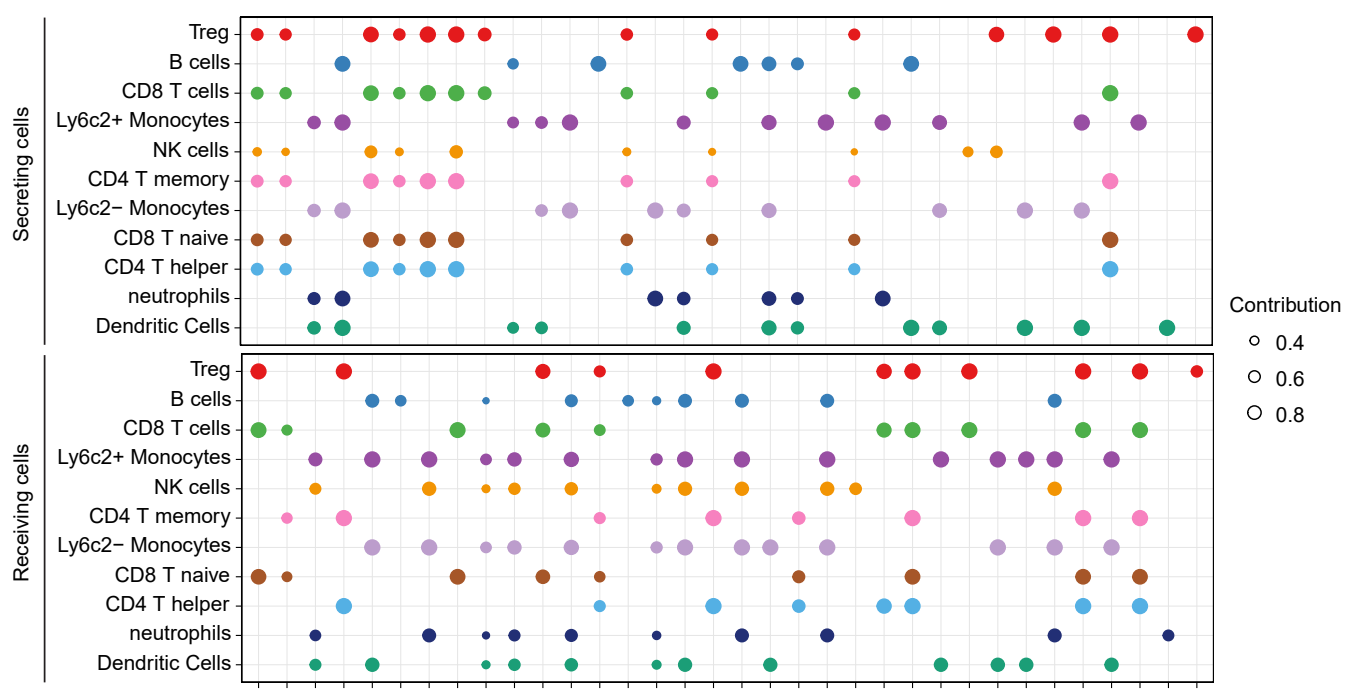

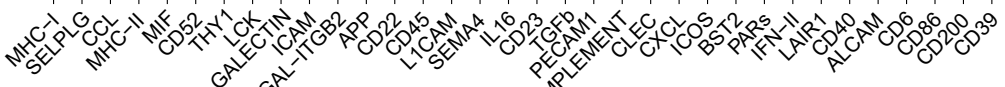

D

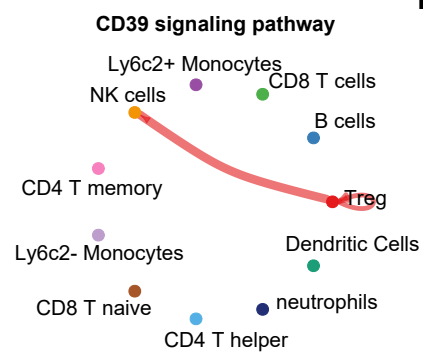

H
E



F



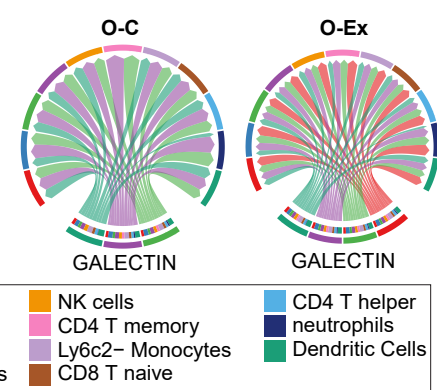

G
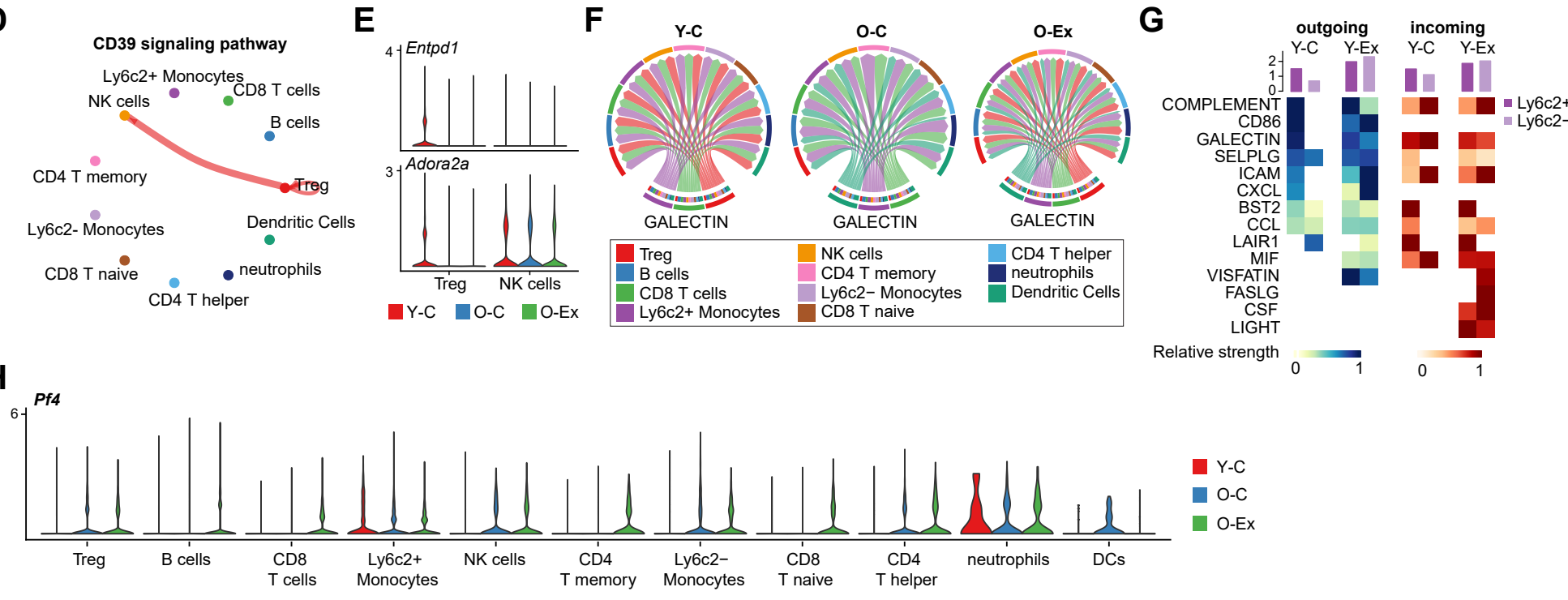
Figure ProRxiv preprint doi: https://doi.org/10.1101/2022.01.12.475145; this version posted January 12,2022 . The copyright holder for this preprint
(which was not certified by peer review) is the author/funder. All riahts reserved. No reuse allowed without permission.

Figure ProRxiv preprint doi: https://doi.org/10.1101/2022.01.12.475145; this version posted January 12,2022 . The copyright holder for this preprint
(which was not certified by peer review) is the author/funder. All rights reserved. No reuse allowed without permission.

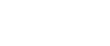

F
C
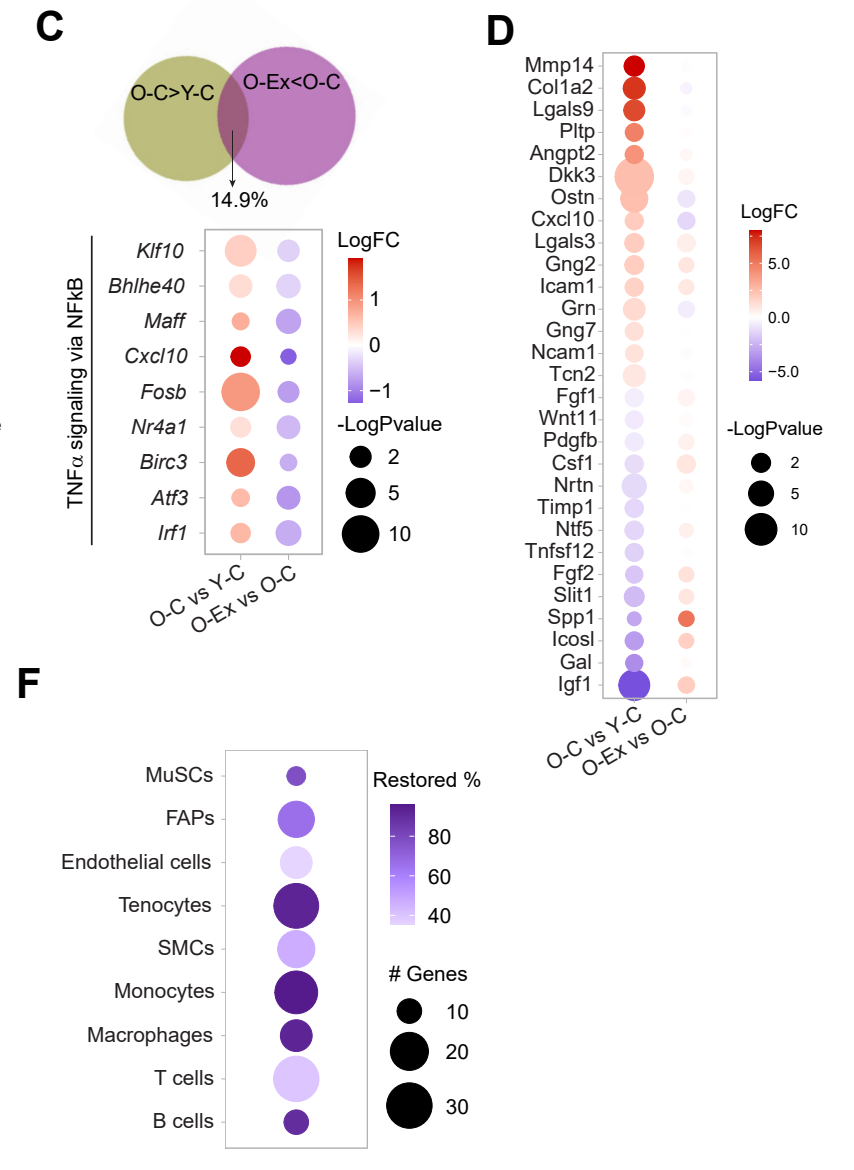

D

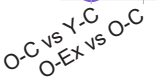
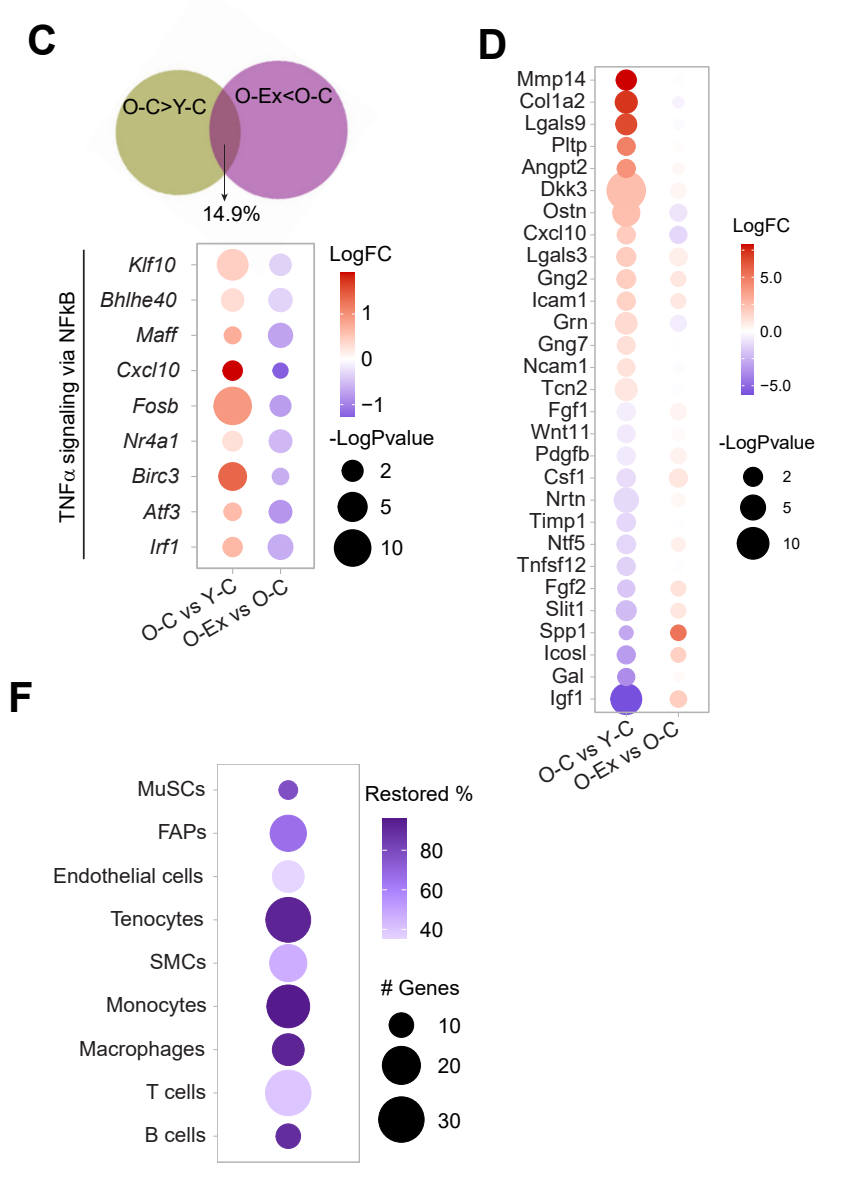
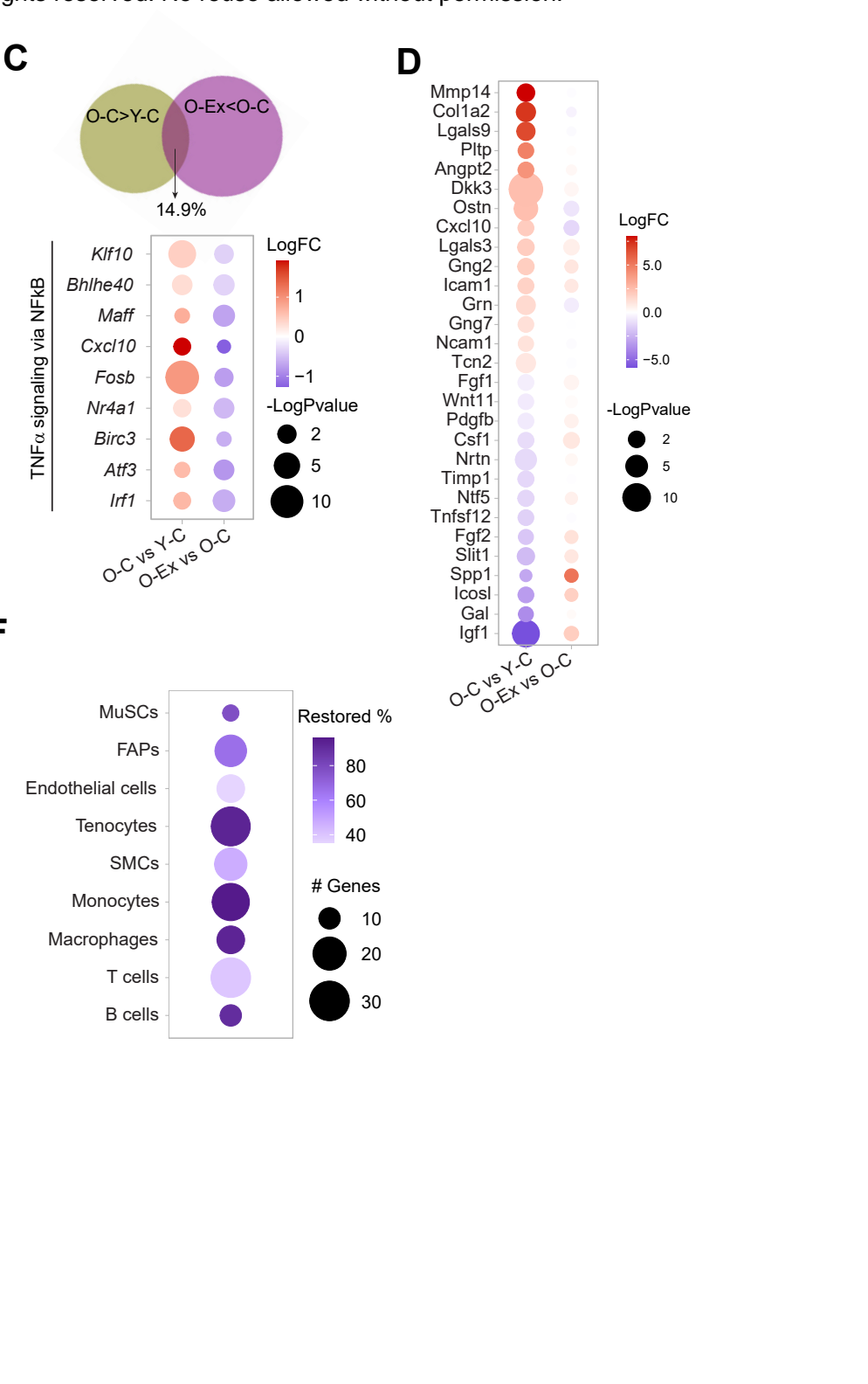
A

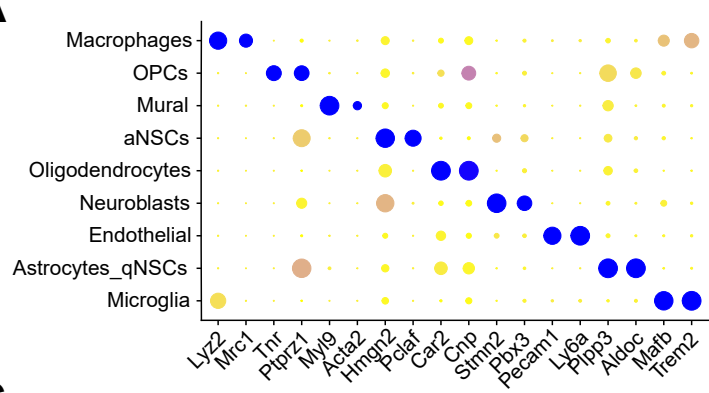

C

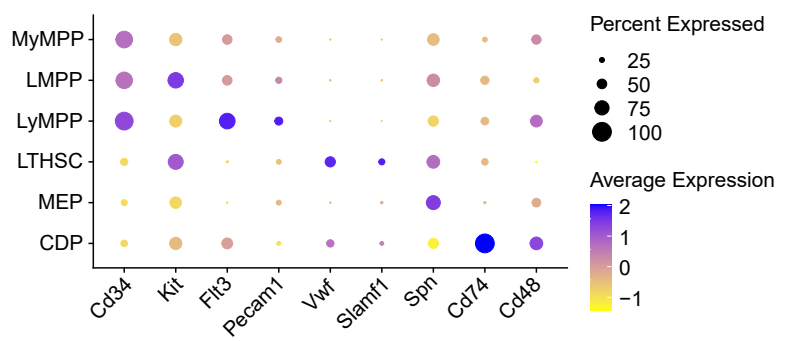

E

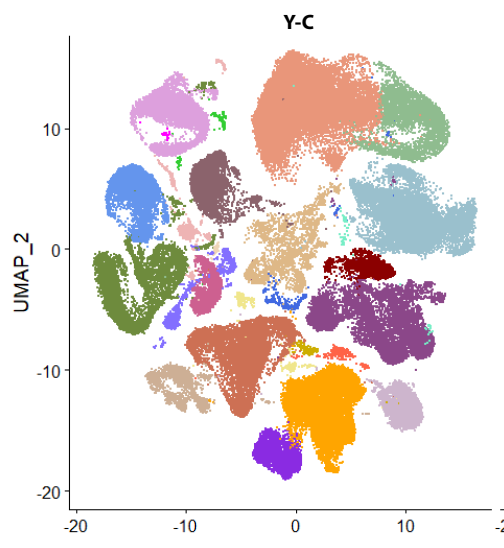

F

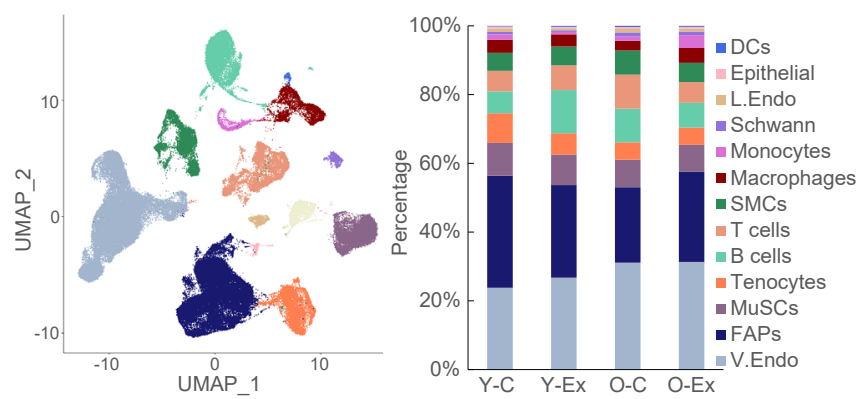

B

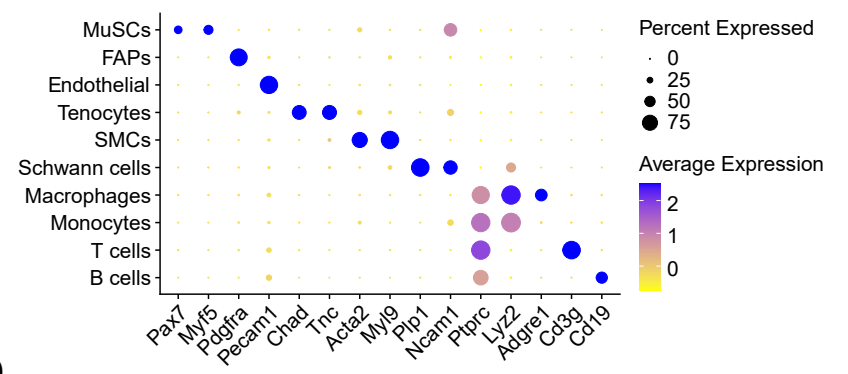

D

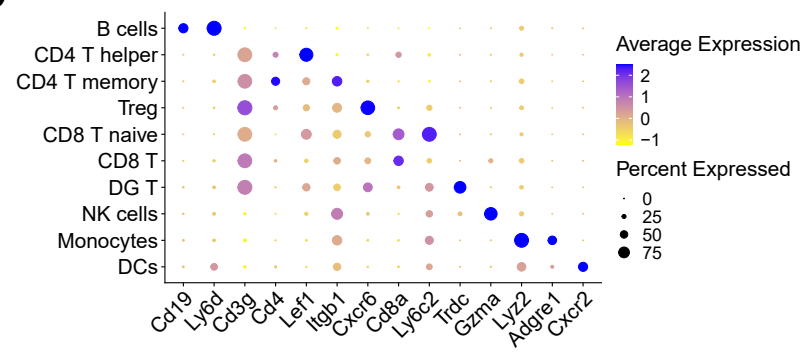

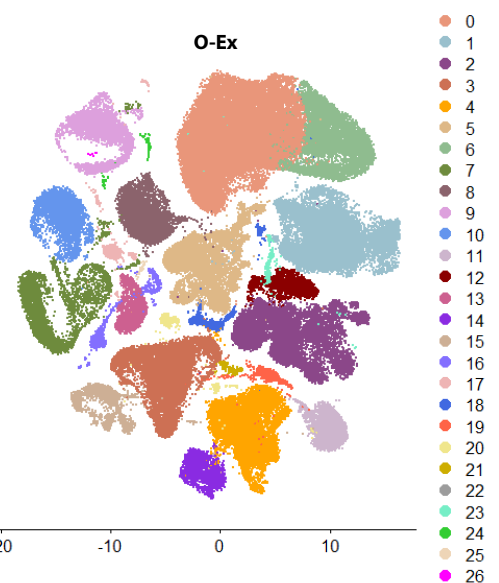

G

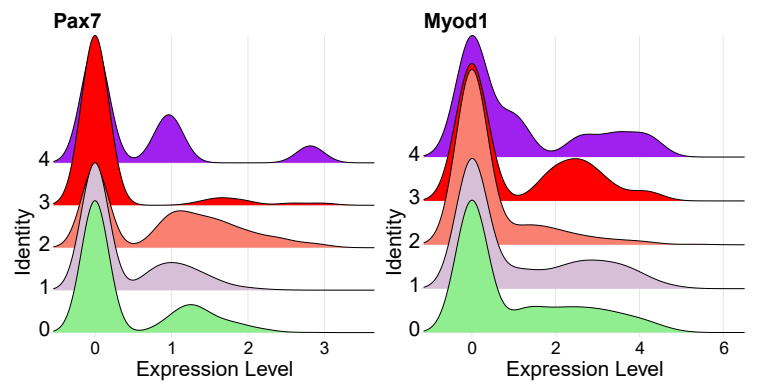


A

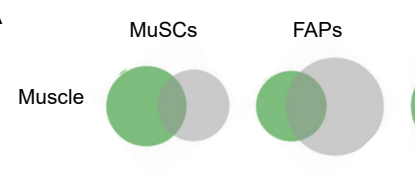

SVZ

Astro/qNSCss blasts/aNSCs
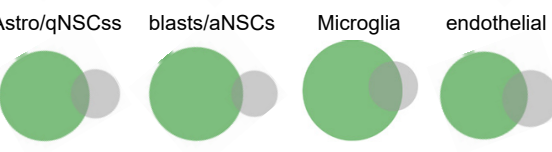

HSCs

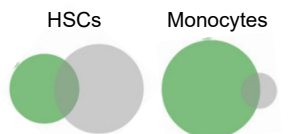

T cells

B cells

Blood

B

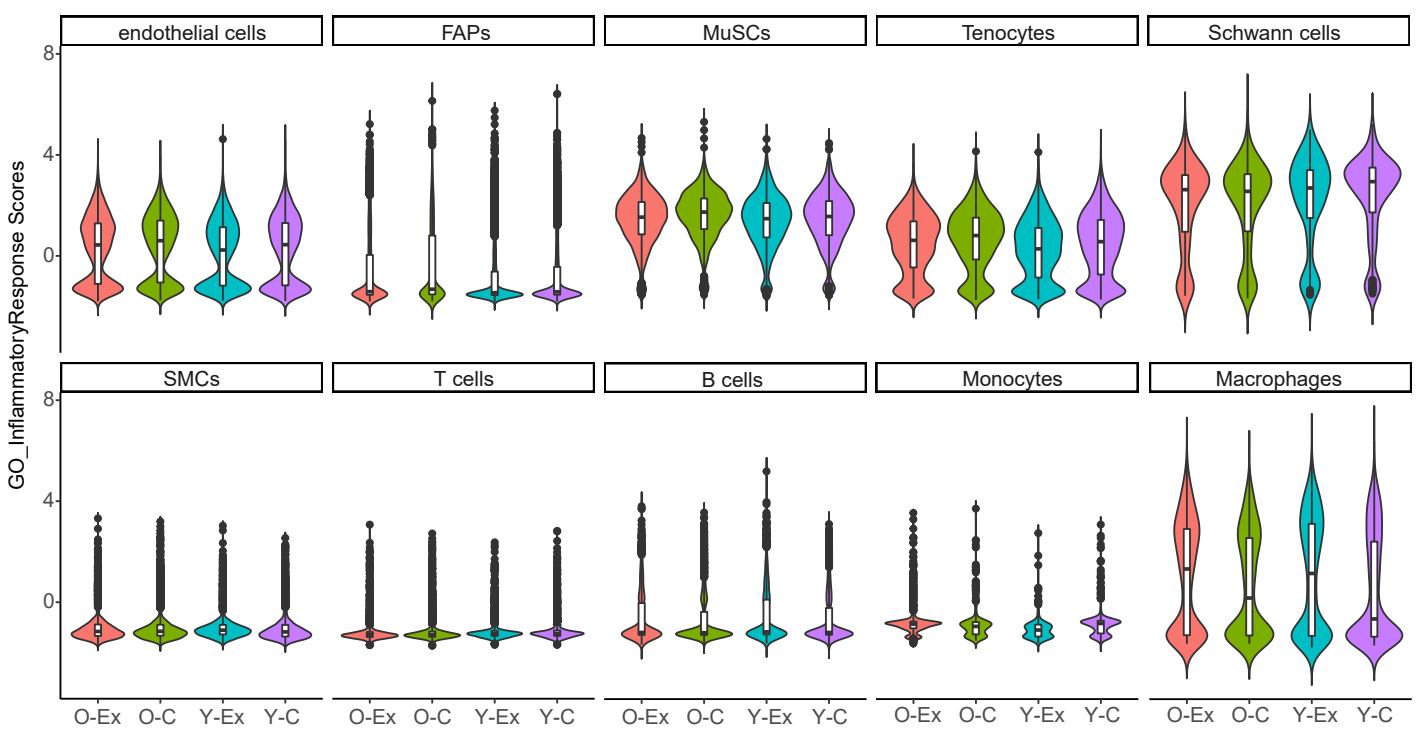

C

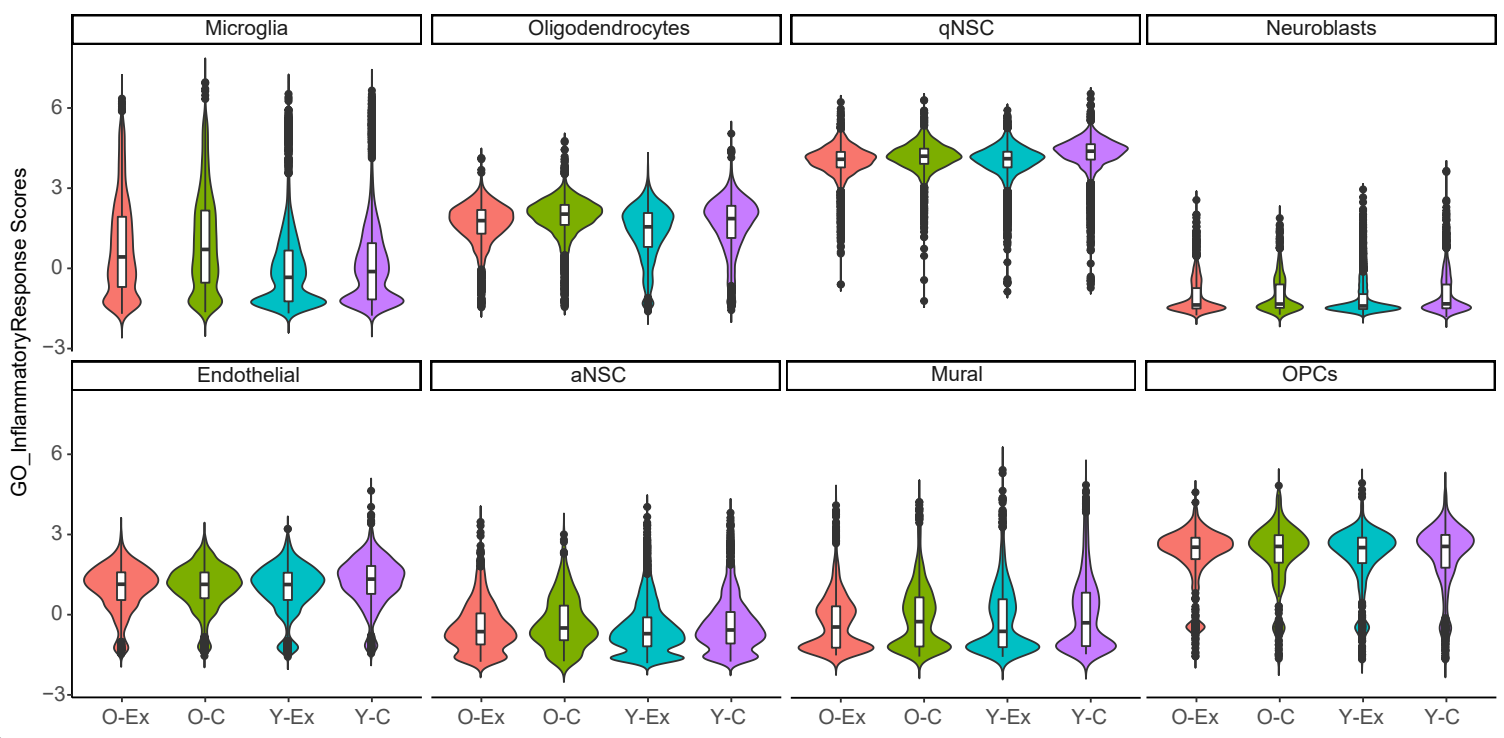

D

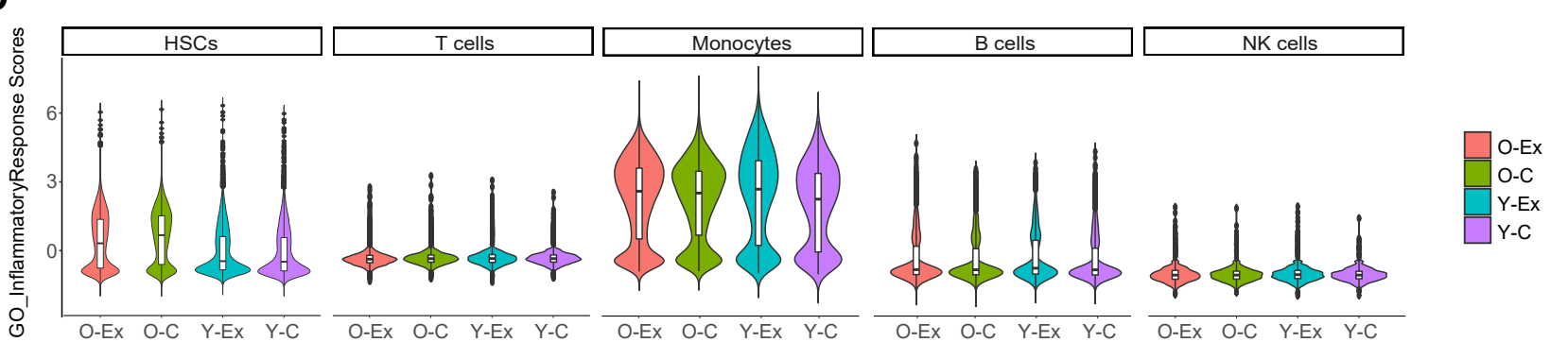

Young Ex-DEGs

Old Ex-DEGs



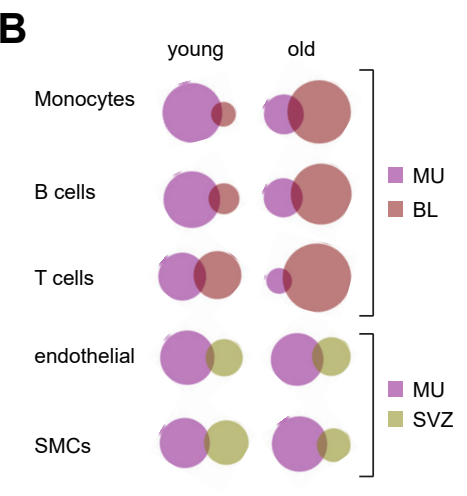


A

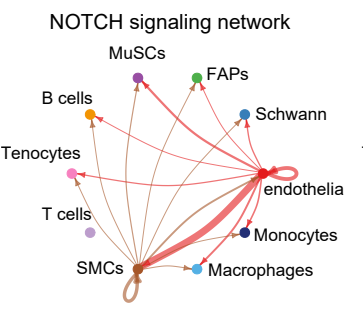

OSM signaling network

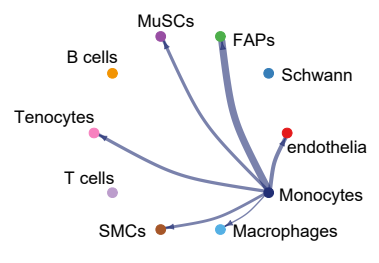

FGF signaling network

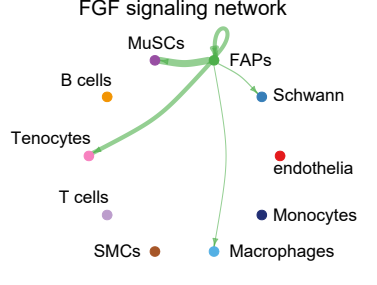

CXCL signaling network

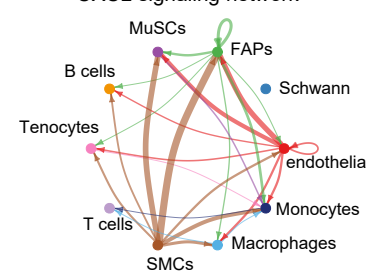

B

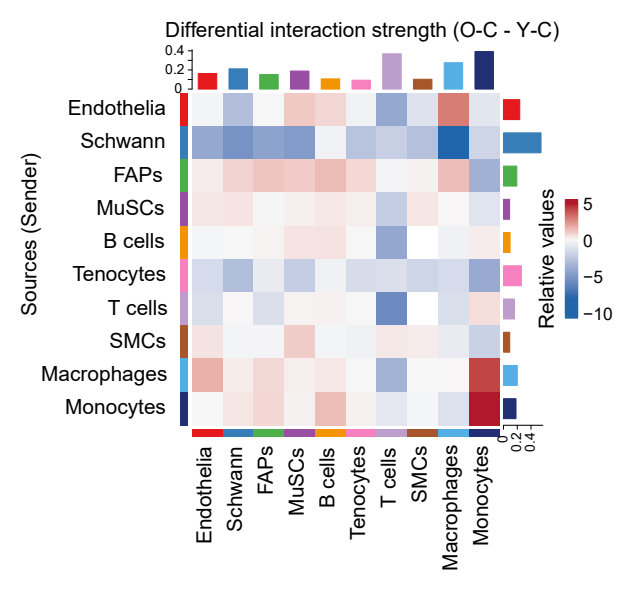

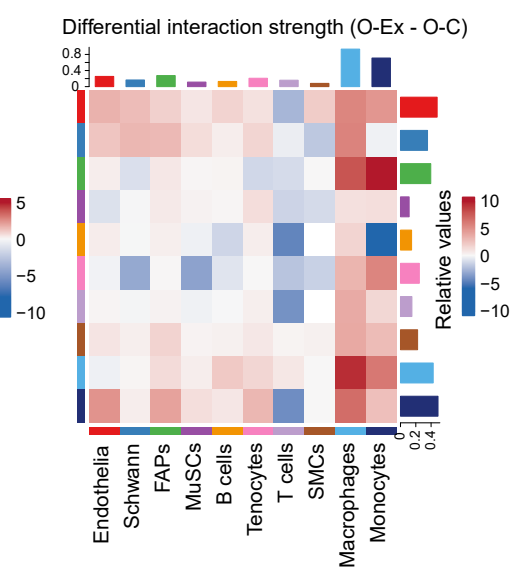

C



$2-1 \quad$ O-C

每

ofx

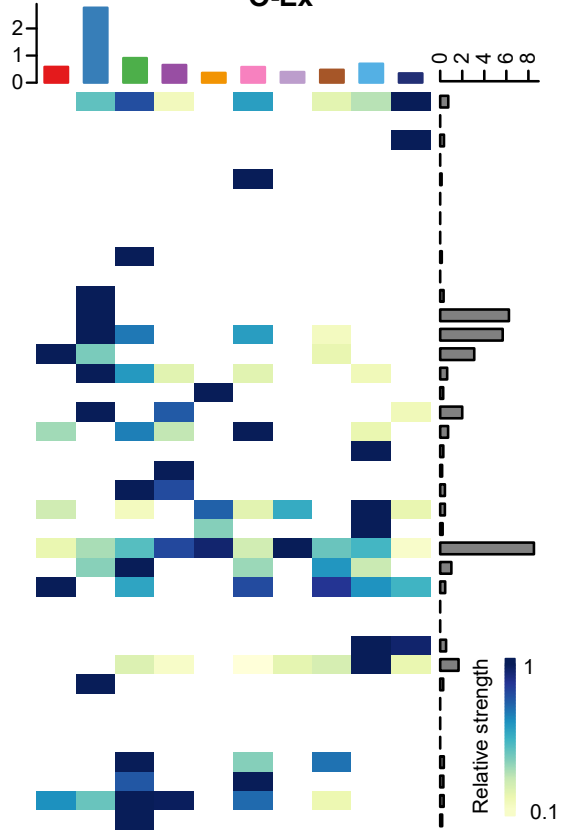

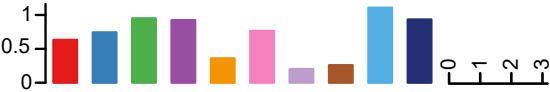



$\square$



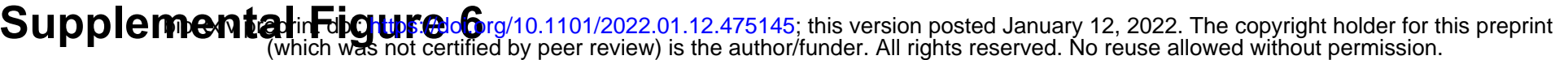

A

D

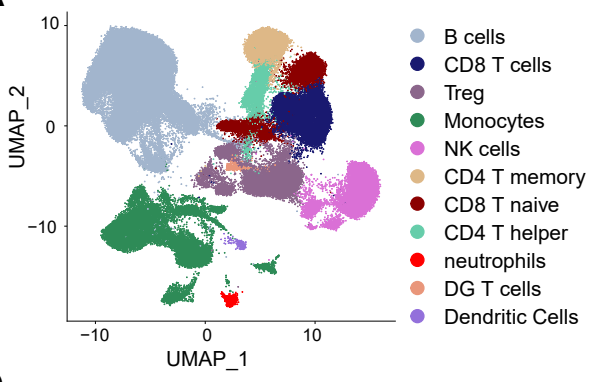

B

\begin{tabular}{|c|c|c|c|c|}
\hline Cell Type & Y-C & Y-Ex & O-C & O-Ex \\
\hline B cells & $49.8 \%$ & $46.0 \%$ & $47.1 \%$ & $40.4 \%$ \\
\hline T cells & $37.2 \%$ & $31.7 \%$ & $33.1 \%$ & $29.6 \%$ \\
\hline NK cells & $8.8 \%$ & $9.9 \%$ & $3.4 \%$ & $7.6 \%$ \\
\hline Monocytes & $4.1 \%$ & $12.0 \%$ & $15.4 \%$ & $21.3 \%$ \\
\hline Dendritic cells & $0.1 \%$ & $0.2 \%$ & $0.3 \%$ & $0.5 \%$ \\
\hline Neutrophils & $0.1 \%$ & $0.3 \%$ & $0.7 \%$ & $0.6 \%$ \\
\hline
\end{tabular}

C

\begin{tabular}{|c|c|c|c|c|}
\hline T cell types & Y-C & Y-Ex & O-C & O-Ex \\
\hline CD4 T memory & $29.2 \%$ & $24.9 \%$ & $7.6 \%$ & $6.3 \%$ \\
\hline Treg & $25.7 \%$ & $39.7 \%$ & $22.4 \%$ & $34.1 \%$ \\
\hline CD8 T Naïve & $19.2 \%$ & $16.3 \%$ & $21.9 \%$ & $12.7 \%$ \\
\hline CD8 T & $18.5 \%$ & $13.3 \%$ & $37.1 \%$ & $35.3 \%$ \\
\hline CD4 T helper & $7.0 \%$ & $5.4 \%$ & $9.9 \%$ & $9.6 \%$ \\
\hline DG T & $0.3 \%$ & $0.5 \%$ & $1.1 \%$ & $2.0 \%$ \\
\hline
\end{tabular}

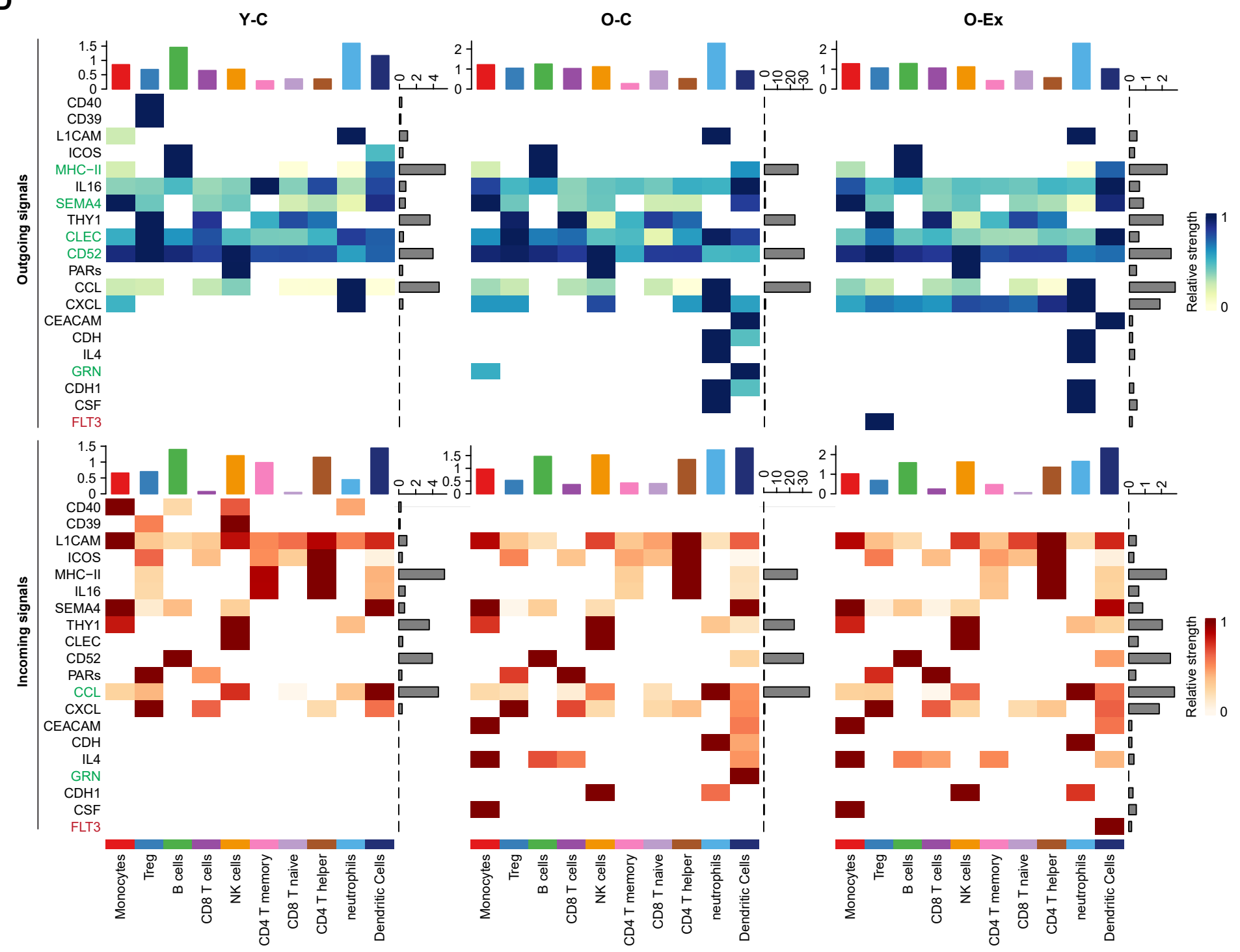


A

D

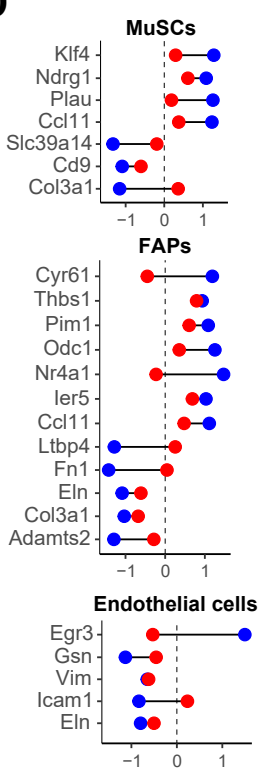

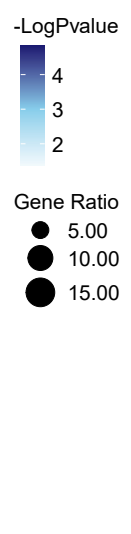

B

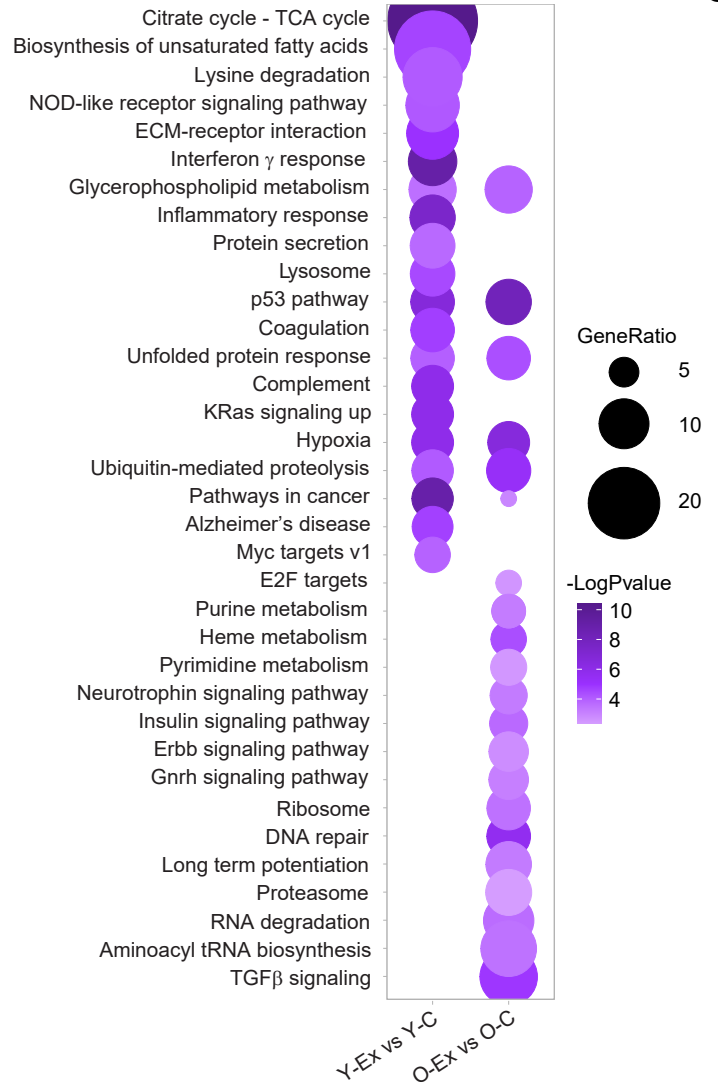

C

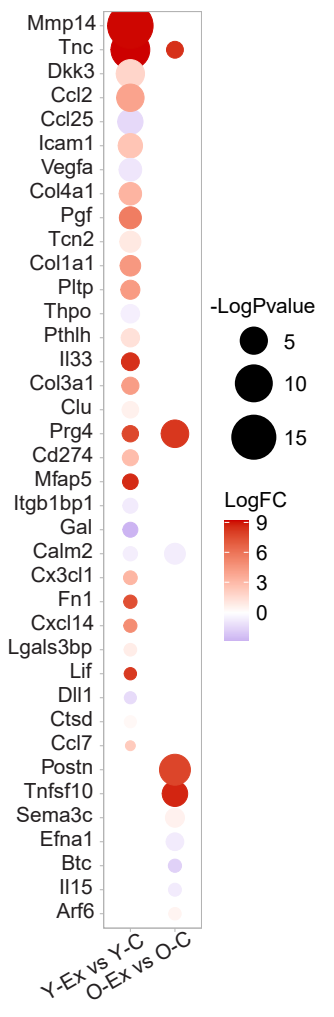

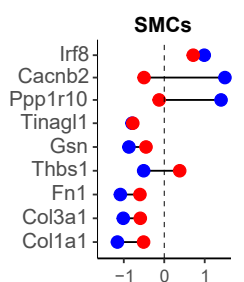

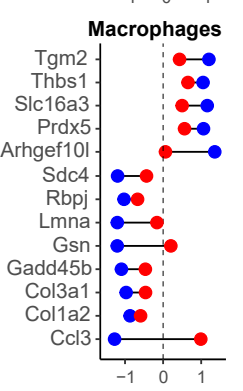



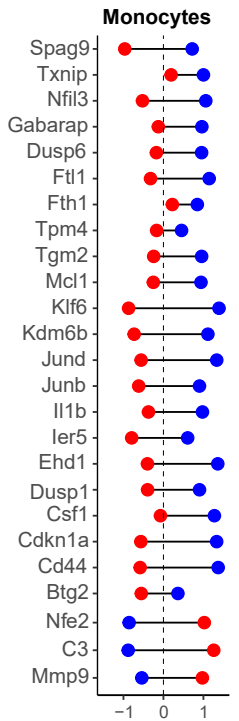

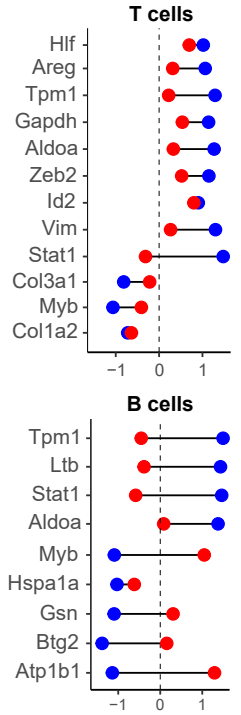

